\title{
TOTAL LINKING NUMBER MODULES
}

\author{
OZIRIDE MANZOLI NETO
}

\begin{abstract}
Given a codimension two link $L$ in a sphere $S^{k}$ with complement $X=S^{k}-L$, the total linking number covering of $L$ is the covering $\hat{X} \rightarrow X$ associated to the surjection $\pi_{1}(X) \rightarrow Z$ defined by sending the meridians to 1. The homology $H_{*}(\hat{X})$ define weaker invariants than the homology of the universal abelian covering of $L$.

The groups $H_{i}(\hat{X})$ are modules over $Z\left[t, t^{-1}\right]$ and this work gives an algebraic characterization of these modules for $k \geq 4$ except for the pseudo null part of $H_{1}(\hat{X})$.
\end{abstract}

Introduction and main results. The total linking number covering of a (differentiable or piecewise-linear locally flat) $m$-component, $n$-link $\ell: \bigcup_{i=1} K_{i}^{n} \rightarrow$ $S^{n+2}\left(K_{i}^{n}\right.$ homeomorphic to $\left.S^{n}\right)$ is the covering $\hat{X} \rightarrow X$ of the complement $X=X(\ell)=S^{n+2}-L(L=$ image $\ell)$ associated to the surjective homomorphism $\alpha: \pi_{1}(X) \rightarrow Z$ defined by sending meridians to 1 .

We can see the homomorphism $\alpha$ as the composition

$$
\pi_{1}(X) \stackrel{A}{\rightarrow} H_{1}(X) \underset{\Omega}{\stackrel{\Omega}{\simeq}} Z^{m} \stackrel{\Sigma}{\rightarrow} Z
$$

where $A$ is abelianization, $\Omega$ is an isomorphism defined by choices of meridians and orientations and $\Sigma$ is sum of coordinates.

In case $\ell$ is a knot the covering $\hat{X}$ coincide with the universal abelian covering $\tilde{X} \rightarrow X$.

There is no general characterization at the moment for the modules $H_{i}(\tilde{X})$.

The aim of this work is the characterization of the collection $\left\{H_{i}(\hat{X})\right\}$ as $Z[Z]$ modules for $n \geq 2$.

The work is presented in four sections. The first contains mostly results on finitely generated modules over $Z[Z]$ (also denoted $\Lambda$ or $Z\left[t, t^{-1}\right]$ ), the second is devoted to finding and selecting properties and relations for the family $\left\{H_{i}(\hat{X})\right\}$ which are suitable for its characterization.

The third section contains the realization work and in the fourth section we find some applications.

All $\Lambda$-modules considered are finitely generated. The conjugate $\bar{M}$ of a $\Lambda$-module $M$ is the same group $M$ with the conjugate action $\lambda\left(t, t^{-1}\right) \cdot \bar{m}=\overline{\lambda\left(t^{-1}, t\right) \cdot m}$.

$[M]$ denotes the stable class of a $\Lambda$-module where $M_{1}$ equivalent to $M_{2}\left(M_{1} \sim\right.$ $M_{2}$ ) means that there are free $\Lambda$-modules $A_{1}$ and $A_{2}$ such that $M_{1} \oplus A_{1} \simeq M_{2} \oplus A_{2}$.

We denote by $T(M)$ the $\Lambda$-torsion part of $M, F T(M)=M / T(M)$ the torsion free part of $M$ and $Q(\Lambda)$ the field of fractions of $\Lambda$.

Received by the editors September 22, 1986.

1980 Mathematics Subject Classification (1985 Revision). Primary 57Q45, 57R40; Secondary $57 \mathrm{M} 25$. 
The pseudo null submodule of $M$ which is defined by $\{m \in M$ such that $\operatorname{Ann}(m)$ is not contained in any principal ideal $\}$ is denoted by $N(M)$ and $N / N(M)$ the pseudo null free part of $M$ is denoted $F N(M)$.

A $\Lambda$-module is said to be of type $K$ if multiplication by $(t-1)$ is an isomorphism of $M$ or equivalently $M \otimes_{\Lambda} Z=0$.

Type $K$ modules are torsion modules, see [L.1, Corollary (1.3)].

The homotopy groups of $X(\ell)$ particularly $\pi_{1}(X(\ell))$ are related to the modules $H_{i}(\hat{X})$ and the characterization of $\pi_{1}(X)$ given by Kervaire $[\mathbf{K}]$ is used in the following sections.

The characterization of the link group $\left(\pi_{1}(X(\ell))\right.$ in connection with the type of covering we are studying suggests the concept of $m$-admissible pairs.

A pair $(G, \alpha)$, where $G$ is a group and $\alpha$ is a surjection $\alpha: G \rightarrow Z$ is called $m$-admissible if there is isomorphism $\Omega: H_{1}(G) \simeq Z^{m}$, weight $(G)=m$ and $\alpha$ is the composition $\Sigma \cdot \Omega \cdot A$ where $A$ is abelianization, $\Sigma$ is sum of coordinates of $Z^{m}$.

If $G$ has also a presentation with deficiency $m$, the pair is said to be strongly $m$-admissible.

Notice that Kervaire's results in $[\mathbf{K . 1}]$ show that the pair $\left(\pi_{1}(X), \alpha\right)$ where $\alpha$ defines the covering $\hat{X} \rightarrow X$ is a $m$-admissible pair and that every $m$-admissible pair $(G, \alpha)$ with $H_{2}(G)=0$ can be obtained in this way.

Using the "trace function" $x: Q(\Lambda) / \Lambda \rightarrow Q$ defined by Trotter in $[\mathbf{T}]$ and a pairing $\langle\rangle:, F \times F \rightarrow Q(\Lambda) / \Lambda$ which is conjugate linear and $\varepsilon$ Hermitian we get by composition a pairing $\langle,\rangle_{x}: F \otimes_{Z} Q \times F \otimes_{Z} Q \rightarrow Q$ which is $Q$-linear and $(-\varepsilon)$-symmetric.

We now state the main results of this work.

THEOREM (A). Let $\ell: \bigcup_{i=1}^{m} K_{i}^{n} \rightarrow S^{n+2}$ a link and $\alpha=\left(\sum \circ \Omega \circ A\right): \pi_{1}(X(\ell))$ $\rightarrow Z$ where $A$ is abelianization, $\Omega$ is isomorphism defined by choices of meridians and orientations, and $\Sigma$ is sum of coordinates of $Z^{m}$.

Denote by $\hat{X}(\ell)$ the covering associated to the kernel of $\alpha$ and $B_{i}=H_{i}(\hat{X}(\ell))$ then $\left(\pi_{1}(X(\ell)), \alpha\right)$ is an $m$-admissible pair and we have the following properties:

(a) $\operatorname{rank}_{\Lambda} B_{1}=(m-1)$,

(b) $T\left(B_{1}\right), B_{2}, \ldots, B_{n}$ are type $K$.

(c) $N\left(\bar{B}_{q}\right) \simeq \operatorname{Ext}_{\Lambda}^{2}\left(N\left(B_{n-q}\right), \Lambda\right)$ and $F N\left(\bar{B}_{q}\right) \simeq \operatorname{Ext}_{\Lambda}^{1}\left(F N\left(B_{n+1-q}\right), \Lambda\right)$ for $2 \leq$ $q \leq n-1$,

(d) $N\left(\bar{B}_{1}\right) \simeq \operatorname{Ext}_{\Lambda}^{2}\left(N\left(B_{n-1}\right), \Lambda\right)$ and $F N\left(T\left(\bar{B}_{1}\right)\right) \simeq \operatorname{Ext}_{\Lambda}^{1}\left(F N\left(B_{n}\right), \Lambda\right)$,

(e) $\bar{B}_{n} \simeq \operatorname{Ext}_{\Lambda}^{1}\left(F N\left(B_{1}\right), \Lambda\right)$,

(f) The stable class $\left[F T\left(\bar{B}_{1}\right)\right]$ determines and is determined by the isomorphism class of $\operatorname{Ext}_{\Lambda}^{2}\left(N\left(B_{n}\right), \Lambda\right)$,

(g) $B_{1} \otimes_{\Lambda} Z \simeq Z^{m-1}$,

(h) The Blanchfield pairing $\langle\rangle:, F N\left(T\left(B_{q}\right)\right) \times F N\left(T\left(B_{n+1-q}\right)\right) \rightarrow Q(\Lambda) / \Lambda$ is defined for $1 \leq q \leq(n+1)$. It is conjugate linear, nonsingular and in case $n=2 q-1$ it is $(-1)^{q+1}-$ Hermitian.

(i) The Levine pairing [, ] : $N\left(B_{q}\right) \times N\left(B_{n-q}\right) \rightarrow Q / Z$ is defined for $1 \leq q \leq$ $(n-1)$. It is $Z$-linear, conjugate selfadjoint, nonsingular and in case $n=2 q$ it is symmetric.

(j) If $n=3$, the index of $\langle,\rangle_{x}:\left(F N\left(T\left(B_{2}\right)\right) \otimes_{Z} Q\right) \times\left(F N\left(T\left(B_{2}\right)\right) \otimes_{Z} Q\right) \rightarrow Q$ is a multiple of 16 . 
(k) $B_{1} \simeq H_{1}\left(\pi_{1}(X(\ell))\right)$,

(l) The Hopf homomorphism $\rho: B_{2} \rightarrow H_{2}\left(\pi_{1}(\hat{X}(\ell))\right)$ is surjective. (Here $\pi_{1}(\hat{X}(\ell))$ is identified with the kernel of $\alpha=\Sigma \cdot \Omega \cdot A$.)

These results extend those known for knots $(m=1)$ and there are similar results for classical links $(n=1)$ which are not so complete.

THEOREM (B). Let $n \geq 2,(G, \alpha)$ an $m$-admissible pair and $\left(B_{2}, B_{3}, \ldots, B_{n}\right)$ a sequence of $\Lambda$-modules of type $K$; denote by $\hat{G}$ the kernel of $\alpha$ and $B_{1}=H_{1}(\hat{G})$.

Let $\rho: B_{2} \rightarrow H_{2}(\hat{G})$ be a surjection and suppose the collection $\left(B_{1}, B_{2}, \ldots, B_{n}\right)$ satisfies the necessary conditions $(\mathrm{c}),(\mathrm{d}),(\mathrm{e}),(\mathrm{f}),(\mathrm{g}),(\mathrm{h}),(\mathrm{i})$ and $(\mathrm{j})$ of Theorem A. Suppose further that we have the (not necessary) conditions.

(1) $B_{2}$ has a pseudo null free summand $F_{2}$ such that $\rho\left(F_{2}\right)=H_{2}(\hat{G})$.

(2) If $n=2, G$ has a presentation of the form $\left|x_{1}, x_{2}, \ldots, x_{k+m} ; r_{1}, r_{2}, \ldots, r_{k}\right|$ with $\left\langle x_{k+1}, \ldots, x_{k+m}\right\rangle=G$ and the relations $r_{j} j=1,2, \ldots, k$, satisfy the conditions, $r_{j}\left(x_{1}, x_{2}, \ldots, x_{k}, 1,1, \ldots, 1\right)=x_{j}$.

Then, there is a link $\ell: \bigcup_{i=1}^{m} S_{i}^{n} \rightarrow S^{n+2}$ with $\pi_{1}(X(\ell))=G$ and if we denote by $\hat{X}(\ell)$ the covering associated to the kernel of $\alpha$, we have for $j=1,2, \ldots, n$, $N\left(B_{j}\right) \simeq N\left(H_{j}(\hat{X}(\ell))\right)$ and $F N\left(B_{j}\right)=F N\left(H_{j}(\hat{X}(\ell))\right)$, and for $i$ such that $1 \leq$ $i \leq[n / 2]$ or $i=n$ we have $B_{i} \simeq H_{i}(\hat{X}(\ell))$.

If we are looking for links of homotopy spheres instead of standard spheres the condition (2) can be eliminated.

THEOREM (C). Let $n \geq 2$ and $\left(B_{1}, B_{2}, \ldots, B_{n}\right)$ a sequence of $\Lambda$-modules satisfying the necessary conditions $(\mathrm{a})$ to $(\mathrm{j})$ of Theorem $\mathrm{A}$.

Suppose further that we have the (not necessary) condition $N\left(B_{1}\right)=0$.

Then there is a link $\ell: \bigcup_{i=1}^{m} S_{i}^{n} \rightarrow S^{n+2}$ such that $N\left(B_{j}\right) \simeq N\left(H_{j}(\hat{X}(\ell))\right)$ and $F N\left(B_{j}\right) \simeq F N\left(H_{j}(\hat{X}(\ell))\right)$ for $j=1,2, \ldots, n$ and $B_{i} \simeq H_{i}(\hat{X}(\ell))$ for $1 \leq i \leq$ $[n / 2]$ and $i=n$.

These are the main results of this work which was presented in partial fulfillment of the requirements for the Ph.D. degree to the Graduate School of Arts and Sciences at Brandeis University. It was developed under the direction of Professor Jerome Levine whom the author wishes to thank.

I would like to thank also the CNPq-Conselho Nacional de Pesquisas (Brasil), the ICMSC, USP, Instituto de Ciências Matemáticas de Sao Carlos (Brasil) and FAPESP-Fundação de Amparo a Pesquisa do Estado de Sao Paulo for the financial support.

1. Algebraic preliminaries. Most of this section is well known and is inserted here for convenience. The basic references are [B.1 and M.1].

Some notation was established in the introduction.

$\Lambda$ is a Krull domain, the height one primes are principal and the maximal primes are of the form $(p, g(t))$ where $p$ is a prime integer and $g(t)$ is a polynomial which becomes irreducible when reduced to $(Z / p Z)\left[t, t^{-1}\right]$.

The set of associated primes of a $\Lambda$-module $M$ is denoted $\operatorname{Ass}(M)$, the set of primes of $\Lambda$ and maximal primes are denoted respectively by $\operatorname{Spec}(\Lambda)$ and $\Omega(\Lambda)$ and the support of $M$ is denoted by $\operatorname{Supp}(M)$. 
Every projective $\Lambda$-module is free, see [S.1].

We keep some of the notation of $[\mathbf{L . 1}] ; e^{i}(M)=\operatorname{Ext}_{\Lambda}^{i}(M, \Lambda), t(M)=Z$-torsion part of $M, f(M)=M / t(M)=$ the $Z$-torsion free part of $M$ and $M^{*}=\operatorname{Hom}_{\Lambda}(M, \Lambda)$.

It is easy to check that a $\Lambda$-module $M$ is pseudo null (definition in the introduction) if and only if $M_{p}$, the localization at $p$, is zero for all height one primes $p$.

Proposition (1.1). A $\Lambda$-module $M$ is pseudo null iff $M$ is finite.

If $M$ is finite, we have $\operatorname{Ass}(M) \subset \Omega(\Lambda)$, but minimal primes of $\operatorname{Supp}(M)$ are in $\operatorname{Ass}(M)$ to $\operatorname{Supp}(M)=\operatorname{Ass}(M)$. Then $M_{p}=0$ for all height one primes, that is, $M$ is pseudo null.

If $M$ is pseudo null, any submodule and any quotient of $M$ is pseudo null.

We have $0 \supset M_{0} \supset M_{1} \supset \cdots \supset M_{n}=M$ with $M_{i+1} / M_{i} \simeq \Lambda / p_{i}$ and $\left\{p_{i}\right\}=$ $\operatorname{Ass}\left(M_{i+1} / M_{i}\right) \subset \Omega(\Lambda)$ since it is pseudo null.

So, each factor is finite because of the form of the maximal ideals of $\Lambda$ and so $M$ is finite.

Proposition (1.2). If $T$ is a $\Lambda$ torsion module then $e^{1}(T) \simeq \operatorname{Hom}_{\Lambda}(T, Q(\Lambda) / \Lambda)$ and for $N$ pseudo null, $e^{1}(N)=0$.

Let $\cdots \rightarrow \operatorname{Hom}(T, Q(\Lambda)) \rightarrow \operatorname{Hom}(T, Q(\Lambda) / \Lambda) \rightarrow e^{1}(T) \rightarrow \operatorname{Ext}_{\Lambda}^{1}(T, Q(\Lambda)) \rightarrow \cdots$ be the long exact sequence of Ext derived from $0 \rightarrow \Lambda \rightarrow Q(\Lambda) \rightarrow Q(\Lambda) / \Lambda \rightarrow 0$.

Since $Q(\Lambda)$ is injective and $T$ is torsion, we have $\operatorname{Hom}(T, Q(\Lambda))$ and $\operatorname{Ext}_{\Lambda}^{1}(T, Q(\Lambda))$ both zero and so $e^{1}(T) \simeq \operatorname{Hom}_{\Lambda}(T, Q(\Lambda) / \Lambda)$.

For the second part of the proposition, we prove that $\operatorname{Hom}_{\Lambda}(N, Q(\Lambda) / \Lambda)=0$.

Let $\Phi: N \rightarrow Q(\Lambda) / \Lambda$ and write $\Phi(x)=[\lambda(t) / \mu(t)]$ where the brackets mean residue class modulo $\Lambda$.

$N$ is pseudo null so let $\operatorname{Ann}(x)=q_{1} \cap q_{2} \cap \cdots \cap q_{n}$ be a primary decomposition of $\operatorname{Ann}(x)$; here each $q_{i}$ is $m_{i}$-primary where $m_{i}=\left(p_{i}, g_{i}(t)\right) \in \Omega(\Lambda)$.

For each $i$, there is an integer $n_{i} \in Z^{+}$, minimal, such that $p_{i}^{n_{i}} \subset q_{i}$ and so, for $m=\pi p_{i}^{n_{i}}$ we have $m x=0$. Then we can put $\Phi(x)=[\Phi(t) / m], \Phi(t) \in \Lambda$ and we can suppose $\phi(t) \in Z[t]$.

$\operatorname{Ann}(x)$ is of height two so for any prime $p$ of $Z$, there is a polynomial $g_{p}(t) \notin(p) \in$ $\operatorname{Spec}(\Lambda)$ such that $g_{p}(t) x=0$; we obtain polynomials $h_{i}(t) \notin\left(p_{i}\right)$ for $i=1,2, \ldots, n$ such that $h_{i}(t) x=0$.

We have then $0=\Phi\left(h_{i}(t) x\right)=\left[h_{i}(t) \phi(t) / m\right]$, that is, $h_{i}(t) \phi(t) \in(m) \subset\left(p_{i}^{n_{i}}\right)$ and so $\phi(t) \in\left(p_{i}^{n_{i}}\right), i=1,2, \ldots, n$. Therefore, $[\phi(t) / m]=0 \in Q(\Lambda) / \Lambda$, that is, $\Phi \equiv 0$.

If $N(M)=0$ we say that the $\Lambda$-module $M$ is pseudo null free and it is equivalent to say that every prime in $\operatorname{Ass}(M)$ has height zero or one.

Proposition (1.3). If $M$ is pseudo null free, then $\operatorname{Ext}_{\Lambda}^{i}(M, P)=0$ for all modules $P$ and all $i \geq 2$.

We have $\operatorname{Ass}(M) \cap \Omega(\Lambda)=\varnothing$, so for a maximal ideal $m$ of $\Lambda$, $\operatorname{depth}_{\Lambda_{m}}\left(M_{m}\right) \geq 1$ and then $1 \leq \operatorname{depth}_{\Lambda_{m}}\left(M_{m}\right)=\operatorname{dim} \Lambda_{m}-$ proj. $\operatorname{dim}\left(M_{m}\right)$ by a result of Auslander and Buchsbaum; see [M.1, p. 114].

From this we get that $1 \leq 2-$ proj. $\operatorname{dim}\left(M_{m}\right)$ or proj. $\operatorname{dim}\left(M_{m}\right) \leq 1$ and so proj. $\operatorname{dim} M=\sup _{m \in \Omega(\Lambda)}\left\{\right.$ proj. $\left.\operatorname{dim}\left(M_{m}\right)\right\} \leq 1$ which means that $\operatorname{Ext}_{\Lambda}^{i}(M, P)=0$ for all $P$ and $i \geq 2$. 
PROPOSITION (1.4). Let $M$ be a $\Lambda$-module. Then

(a) $\operatorname{Hom}_{\Lambda}(F N(M), Q(\Lambda) / \Lambda) \simeq \operatorname{Hom}_{\Lambda}(M, Q(\Lambda) / \Lambda)$,

(b) $e^{1}(F N(M)) \simeq e^{1}(M)$,

(c) $e^{2}(N(M)) \simeq e^{2}(M) \simeq \operatorname{Ext}_{\Lambda}^{1}(M, Q(\Lambda) / \Lambda) \simeq \operatorname{Ext}_{\Lambda}^{1}(N(M), Q(\Lambda) / \Lambda)$.

(d) For $i \geq 2$, $\operatorname{Ext}_{\Lambda}^{i}(M, Q(\Lambda) / \Lambda)=0=e^{i+1}(M)$,

(e) $e^{1}(T(M)) \simeq \operatorname{Hom}_{\Lambda}(T(M), Q(\Lambda) / \Lambda) \simeq \operatorname{Hom}_{\Lambda}(F N(T(M)), Q(\Lambda) / \Lambda)$,

(f) $\operatorname{Hom}_{\Lambda}(M, Q(\Lambda)) \simeq \operatorname{Hom}_{\Lambda}(F T(M), Q(\Lambda)) \simeq \operatorname{Hom}_{\Lambda}\left(F T(M)^{* *}, Q(\Lambda)\right)$,

(g) Cokernel $(t-1): M \rightarrow M \simeq M \otimes_{\Lambda} Z$ (in particular $\left.Z \otimes_{\Lambda} Z \simeq Z\right)$.

(h) If $M$ is of type $K$ then $\operatorname{Tor}_{\Lambda}^{i}(M, Z)=0=\operatorname{Ext}_{\Lambda}^{i}(M, Z)$ for any $i \geq 1$,

(i) $e^{1}(Z) \simeq Z$,

(j) If $T$ is torsion then $\operatorname{Ext}_{\Lambda}^{i}(T, Q(\Lambda))=0$ for every $i$.

The proof can be obtained from the exact sequences $0 \rightarrow \Lambda \rightarrow Q(\Lambda) \rightarrow Q(\Lambda) / \Lambda$ $\rightarrow 0,0 \rightarrow N(M) \rightarrow M \rightarrow F N(M) \rightarrow 0,0 \rightarrow T(M) \rightarrow M \rightarrow F T(M) \rightarrow 0$ or $0 \rightarrow \Lambda \stackrel{(t-1)}{\rightarrow} \Lambda \rightarrow Z \rightarrow 0$ and the sequences derived from them by Tor and Ext.

For the last part one can use the fact that the canonical homomorphism $M \rightarrow$ $M^{* *}$ is a pseudo isomorphism, see [B.1].

PROPOSITION (1.5). If $T$ is a $\Lambda$-torsion module and $x \in T-N(T)$, there is an homomorphism $\phi: T \rightarrow Q(\Lambda) / \Lambda$ such that $\phi(x) \neq 0$.

It is sufficient to prove this for $N(T)=0$.

If $T=\Lambda / p^{n}$ where $p$ is a prime of height one, take $\varphi: \Lambda \rightarrow Q(\Lambda)$ such that $\varphi(1)=q(t) / p^{n}$ where $(q(t), p)=(1)$; the induced map $\phi: \Lambda / p^{r} \rightarrow Q(\Lambda) / \Lambda$ is a monomorphism.

If $T \simeq \bigoplus_{i=1}^{k} \Lambda / p_{i}^{n_{i}}$, just project into some factor and use the above argument.

For the general situation, we use the fact that there is a pseudo-isomorphism $T \stackrel{i}{\rightarrow} \bigoplus_{i=1}^{k} \Lambda / p_{i}^{n_{i}}$ which is a monomorphism because $N(T)=0$.

If we write $W=\bigoplus \Lambda / p_{i}^{n_{i}}$, we have $0 \rightarrow T \stackrel{i}{\rightarrow} W \rightarrow W / T \rightarrow 0$ with $W / T$ pseudo null. The induced $\operatorname{map} \operatorname{Hom}_{\Lambda}(W, Q(\Lambda) / \Lambda) \stackrel{i^{*}}{\rightarrow} \operatorname{Hom}_{\Lambda}(T, Q(\Lambda) / \Lambda)$ is a monomorphism because $\operatorname{Hom}_{\Lambda}(W / T, Q(\Lambda) / \Lambda)=0$.

Taking now $x \in T, x \neq 0, i(x) \neq 0 \in W$ so, from the above, there exists $\phi: W \rightarrow Q(\Lambda) / \Lambda$ such that $\phi(i(x)) \neq 0$, i.e., then $i_{*}(\phi)(x)=(i(x)) \neq 0$ and $i_{*}(\phi)$ satisfies the condition.

Proposition (1.6). For any $\Lambda$-modules $M$ and $P, e^{2}(M)$ and $\operatorname{Ext}_{\Lambda}^{1}(N(M), P)$ are pseudo null.

Since $e^{2}(M) \simeq e^{2}(N(M))$ we can suppose $M$ pseudo null.

We have then $\left[e^{2}(M)\right]_{p}=\operatorname{Ext}_{\Lambda}^{1}\left(M_{p},(Q(\Lambda) / \Lambda)_{p}\right)$ by localizing at the prime $p$.

If $p$ is of height one, then $M_{p}=0$ and so $\left[e^{2}(M)\right]_{p}=0$, that is, $e^{2}(M)$ is pseudo null.

The same proof works for $\operatorname{Ext}_{\Lambda}^{1}(N(M), P)$.

PROPOSITION (1.7). If $M$ is a torsion free $\Lambda$-module, then $e^{1}(M)$ is pseudo null.

$M$ can be embedded in a free module $F$ since $\Lambda$ is a domain.

The long exact sequence of Ext derived from $0 \rightarrow M \rightarrow F \rightarrow F / M \rightarrow 0$ gives us $e^{1}(M) \simeq e^{2}(F / M)$ which is pseudo null by the last proposition. 

free.

Proposition (1.8). If $T$ is a $\Lambda$-torsion module, then $e^{1}(T)$ is pseudo null

Since $e^{1}(T) \simeq e^{1}(F N(T))$, it is sufficient to prove this for $T$ pseudo null free.

We have then $\operatorname{Ass}(T) \cap \Omega(\Lambda)=\varnothing$ and, for each prime $p$,

$$
\left[e^{1}(T)\right]_{p}=\operatorname{Hom}_{\Lambda_{p}}\left(T_{p},\left(Q(\Lambda) / \Lambda_{p}\right) .\right.
$$

If $p$ is such that $T_{p}=0$ we get $\left[e^{1}(T)\right]_{p}=0$.

If $p$ is such that $T_{p} \neq 0$, then $p \in \operatorname{Supp}(T)=\operatorname{Ass}(T)$. Since $T$ is torsion and pseudo null free, the height of $p$ is one and so $\operatorname{Supp}\left(e^{1}(T)\right)$ only has height one primes which means that $e^{1}(T)$ is pseudo null free.

PROPOSITION (1.9). Let $M$ be a $\Lambda$-module. Then there is an exact sequence $0 \rightarrow M^{*} \rightarrow F \rightarrow F / M^{*} \rightarrow 0$ where $F$ is free and $F / M^{*}$ is pseudo null free.

$M^{*}$ is a reflexive module and is a $\Lambda$-lattice in the $Q(\Lambda)$-vector space $V=$ $\operatorname{Hom}_{\Lambda}(M, Q(\Lambda))$; see [B.1].

We obtain then a free $\Lambda$-module $F \subset V$ and $M^{*} \subset F$. Moreover (Theorem 2, p. 50 [B.1]), $\operatorname{Ass}\left(V / M^{*}\right)$ has only primes of height one and so $\operatorname{Ass}\left(F / M^{*}\right)$ has only primes of height one, that is, $F / M^{*}$ is pseudo null free.

COROLlary (1.10). For any $\Lambda$-module $M, M^{*}$ is free.

Taking $0 \rightarrow M^{*} \rightarrow F \rightarrow F / M^{*} \rightarrow 0$ as in the proposition we have $e^{1}\left(M^{*}\right) \simeq$ $e^{2}\left(F / M^{*}\right)=0$ (long exact sequence of Ext).

Taking now a free resolution of $M^{*}, 0 \rightarrow F_{1} \stackrel{d}{\rightarrow} F_{0} \rightarrow M^{*} \rightarrow 0$, we get

$$
0 \rightarrow M^{* *} \rightarrow F_{0}^{*} \stackrel{d^{*}}{\rightarrow} F_{1}^{*} \rightarrow e^{1}\left(M^{*}\right)=0
$$

and so $d^{*}$ is a split epimorphism which gives us that $d$ is a split monomorphism, that is, $M^{*}$ is free.

PROpOSITION (1.11). If $M$ is a $\Lambda$-module and $x \in M-N(M)$, there is $a$ homomorphism $\phi: M \rightarrow Q(\Lambda) / \Lambda$ with $\phi(x) \neq 0$.

We can suppose $N(M)=0$ and $x \neq 0$.

If $M$ is a torsion module this is Proposition (1.5).

If $M$ is torsion free, we have the canonical homomorphism $M \rightarrow M^{* *}$ which is a pseudo-isomorphism, but $M$ is pseudo null free so $M \rightarrow M^{* *}$ is a monomorphism.

The proposition is obvious for $M^{* *}$ because it is free by Corollary (1.10) so it is true for torsion free $M$.

If $M$ is not torsion or torsion free we can find a pseudo-isomorphism $f: M \rightarrow$ $T(M) \oplus F T(M)$ (see Theorem 4, p. 57 of [B.1]), which is again a monomorphism since $M$ is pseudo null free.

The rest of the proof is now obvious.

Corollary (1.12). A $\Lambda$-module $M$ is pseudo null (finite) if and only if $\operatorname{Hom}_{\Lambda}(M, Q(\Lambda) / \Lambda)=0$.

By the Proposition (1.2) the condition is sufficient and the necessary condition is the proposition above. 
PROPOSITION (1.13). If $N$ is a pseudo null module and $0 \rightarrow M \rightarrow F \rightarrow N \rightarrow$ 0 is exact with $F$ free, then $F \simeq M^{*}$.

We have $N^{*}=0=e^{1}(N)$ and so the exact sequence of Ext derived from $0 \rightarrow$ $M \rightarrow F \rightarrow N \rightarrow 0$ gives us $F^{*} \simeq M^{*}$.

Since $F^{*} \simeq F$ we have $F \simeq M^{*}$.

PROPOSITION (1.14). If $M$ is a torsion free $\Lambda$-module, $e^{1}(M)$ is pseudo null.

Since $\Lambda$ is a domain we have an exact sequence $0 \rightarrow M \rightarrow F \rightarrow F / M \rightarrow 0$ with $F$ free.

The long exact sequence of Ext gives us $e^{1}(M) \simeq e^{2}(F / M)$ which is pseudo null by Proposition (1.6).

Proposition (1.15). For any $\Lambda$-module $M$, there is an exact sequence $0 \rightarrow$ $e^{1}(F T(M)) \rightarrow e^{1}(M) \rightarrow e^{1}(T(M)) \rightarrow 0$ and so $e^{1}(M)$ is $\Lambda$-torsion.

The exact sequence of Ext derived from $0 \rightarrow T(M) \rightarrow M \rightarrow F T(M) \rightarrow 0$ gives us the sequence above because $e^{2}(F T(M))=0=(T(M))^{*}$.

The last part follows because $e^{1}(F T(M))$ is pseudo null and so torsion.

Proposition (1.16). For any $\Lambda$-module $M, e^{2}(M) \simeq \operatorname{Hom}_{Z}(N(M), Q / Z)$ in a natural way, that is, $\operatorname{Hom}_{Z}(N(M), Q / Z)$ with the $\Lambda$-structure coming from $N(M)$ is $\Lambda$-isomorphic to $e^{2}(M)$ and the isomorphism is functorial.

We have $e^{2}(M) \simeq e^{2}(N(M))$ and so it is sufficient to consider the case $M$ pseudo null.

Notice that $M$ pseudo null implies $e^{1}(M)=0$ and $M$ finite, and these two conditions are the only ones used in the proof of Proposition (4.2) in [L.1] so the proof follows.

Proposition (1.17). For a $\Lambda$-module $M, e^{2}\left(e^{2}(M)\right) \simeq N(M)$.

We have $e^{2}\left(e^{2}(M)\right) \simeq e^{2}\left(e^{2}(N(M))\right)$ and $e^{1}(N(M))=0=N(M)^{*}$.

From a free resolution of $N(M)$,

$$
0 \rightarrow F_{2} \rightarrow F_{1} \stackrel{d}{\rightarrow} F_{0} \rightarrow N(M) \rightarrow 0
$$

we get a free resolution of $e^{2}(N(M))$,

$$
0 \rightarrow F_{0}^{*} \stackrel{d_{*}}{\rightarrow} F_{1}^{*} \rightarrow F_{2}^{*} \rightarrow e^{2}(N(M)) \rightarrow 0 .
$$

It follows that: $e^{2}\left(e^{2}(M)\right) \simeq e^{2}\left(e^{2}(N(M))\right) \simeq$ cokernel $d^{* *} \simeq$ cokernel $d \simeq$ $N(M)$.

PROPOSITION(1.18). For a $\Lambda$-module $M$, the following statements are equivalent:

(a) $N(M)=0$,

(b) $e^{2}(M)=0$,

(c) homological dimension of $M \leq 1$.

(a) $\Leftrightarrow(\mathrm{b})$ is clear because $e^{2}(M)=e^{2}(N(M))$ and $e^{2}\left(e^{2}(N(M))\right)=N(M)$.

$(c) \Rightarrow(b)$ is obvious.

To prove $(\mathrm{b}) \Rightarrow(\mathrm{c})$, take a free resolution of $M$

$$
0 \rightarrow F_{2} \stackrel{e}{\rightarrow} F_{1} \stackrel{d}{\rightarrow} F_{0} \rightarrow M \rightarrow 0 .
$$


Its dual is

$$
F_{0}^{*} \stackrel{d^{*}}{\rightarrow} F_{1}^{*} \stackrel{e^{*}}{\rightarrow} F_{2}^{*} \rightarrow e^{2}(M)=0
$$

and so $e^{*}$ is a split epimorphism. Then $e$ is a split monomorphism, that is, homological dimension of $M \leq 1$.

Proposition (1.19). For a $\Lambda$-module $M, e^{1}\left(e^{1}(M)\right) \simeq F N(M)$.

We have by Proposition (1.4) that $e^{1}(M) \simeq e^{1}(F N(M))$.

Taking now a free resolution of $F N(M)$ (which is of homological dimension $\leq 1$ by Proposition 1.18) $0 \rightarrow F_{1} \stackrel{d}{\rightarrow} F_{0} \rightarrow F N(M) \rightarrow 0$ and dualizing, we have

$$
0 \rightarrow F N(M)^{*} \rightarrow F_{0}^{*} \stackrel{d_{*}}{\rightarrow} F_{1}^{*} \rightarrow e^{1}(F N(M)) \rightarrow 0
$$

which is a free resolution of $e^{1}(F N(M))$ and so

$$
e^{1}\left(e^{1}(M)\right) \simeq e^{1}\left(e^{1}(F N(M))\right) \simeq \text { cokernel of } d^{* *} \simeq \text { cokernel } d \simeq F N(M) .
$$

It is interesting to note that for a $\Lambda$-module $M$ we have a diagram of implications.

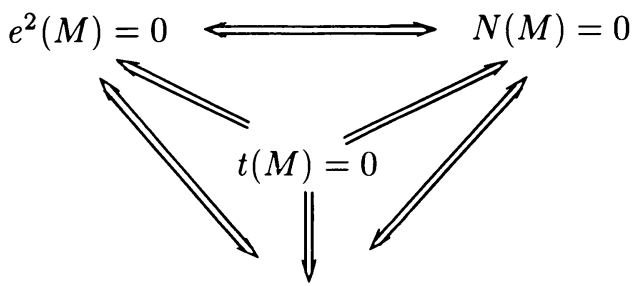

homological dimensional $\quad M \leq 1$

and the one-way arrows can be reversed if $M$ is type $K$.

Proposition (1.20). For a $\Lambda$-module $M, e^{1}\left(e^{2}(M)\right)=0$ and for a $\Lambda$-torsion module $T, e^{2}\left(e^{1}(T)\right)=0$.

$$
\begin{aligned}
e^{1}\left(e^{2}(M)\right) & =e^{1}\left(F N\left(e^{2}(M)\right)\right)=e^{1}(0)=0 \\
e^{2}\left(e^{1}(T)\right) & =e^{2}\left(N\left(e^{1}(T)\right)\right)=e^{2}(0)=0
\end{aligned}
$$

Proposition (1.21). If $F$ is free, $T$ is $\Lambda$-torsion and $0 \rightarrow M \rightarrow F \stackrel{\phi}{\rightarrow} T \rightarrow 0$ is exact, then we can identify $M^{* *}$ with $\phi^{-1}(N(T))$.

We have $e^{1}\left(e^{1}(T)\right)=F N(T)$ by Proposition $(1.19)$, so $0 \rightarrow N(T) \rightarrow T \rightarrow$ $e^{1}\left(e^{1}(T)\right) \rightarrow 0$ exact. 
Since $T^{*}=0$ we have a free resolution $0 \rightarrow F^{*} \rightarrow M^{*} \rightarrow e^{1}(T) \rightarrow 0$ of $e^{1}(T)$ and then we get the commutative diagram below with exact rows and columns.

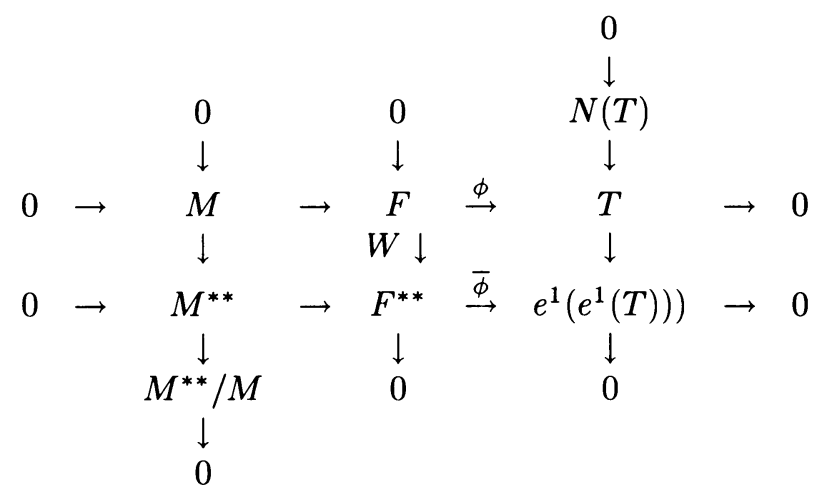

From the snake lemma we have $M^{* *} / M \simeq N(T)$ and $\phi^{-1}(N(T)) \simeq w^{-1}\left(M^{* *}\right)$ which is the identification.

OBSERvations. (a) If $T$ is pseudo null free, we have $w^{-1}\left(M^{* *}\right)=\phi^{-1}(0)=$ image $(M \rightarrow F)$ and $M^{* *} / M=0$, that is $M^{* *}=M$ and so $M$ is free.

(b) If $T=N(T)$, we have $w^{-1}\left(M^{* *}\right)=\phi^{-1}(T)=F$ and so the pseudo null module $T$ is isomorphic to $M^{* *} / M$.

We denote by $[M]$, the stable class of $M$ (see introduction) and by $\{M\}$, the isomorphism class of $M$.

PROPOSITION (1.22). There is a one-to-one correspondence between isomorphism classes of pseudo null $\Lambda$-modules and stable classes of torsion free $\Lambda$-modules given by $\{N\}$ correspond to $[M](\{N\} \leftrightarrow[M])$ if and only if there exists a exact sequence $0 \rightarrow M \rightarrow F \rightarrow N \rightarrow 0$ with $F$ free.

Note that by observation (b) we can always suppose $F=M^{* *}$. Let $\left[M_{1}\right]=\left[M_{2}\right]$, $0 \rightarrow M_{1} \rightarrow M_{1}^{* *} \rightarrow N_{1} \rightarrow 0$ and $0 \rightarrow M_{2} \rightarrow M_{2}^{* *} \rightarrow N_{2} \rightarrow 0$ exact sequences.

We have an isomorphism $\phi: M_{1} \oplus F_{1} \rightarrow M_{2} \oplus F_{2}$ where $F_{1}$ and $F_{2}$ are free and the diagram

$$
\begin{aligned}
& 0 \quad \rightarrow \quad M_{1} \oplus F_{1} \quad \rightarrow \quad M_{1}^{* *} \oplus F_{1}^{* *} \quad \rightarrow \quad N_{1} \rightarrow 0
\end{aligned}
$$

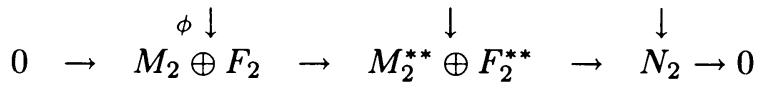

can be completed using maps induced by $\phi$ which are dual isomorphisms so $\left\{N_{1}\right\}=$ $\left\{N_{2}\right\}$.

Let now $\left\{N_{1}\right\}=\left\{N_{2}\right\}$, that is, there is an isomorphism $\phi: N_{1} \rightarrow N_{2}$.

Considering

$$
0 \rightarrow M_{1} \stackrel{w_{1}}{\rightarrow} M_{1}^{* *} \stackrel{\pi_{1}}{\rightarrow} N_{1} \rightarrow 0
$$

and

$$
0 \rightarrow M_{2} \stackrel{w_{2}}{\rightarrow} M_{2}^{* *} \stackrel{\pi_{2}}{\rightarrow} N_{2} \rightarrow 0
$$


we can get diagrams:

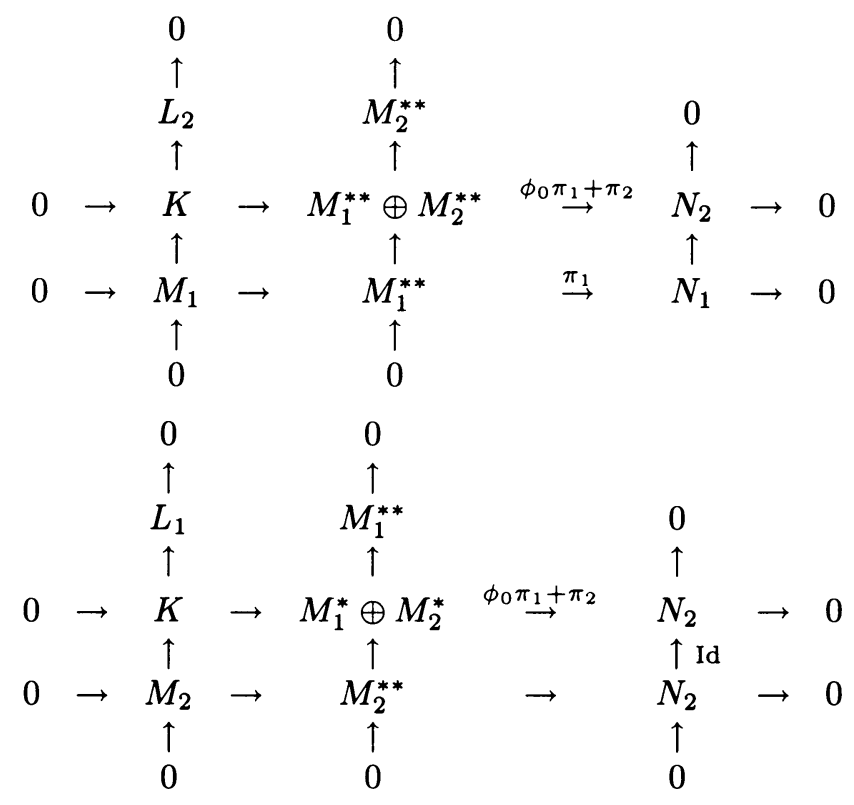

By the snake lemma we get $L_{2} \simeq M_{2}^{* *}$ and $L_{1} \simeq M_{1}^{* *}$, so the first column of each diagram splits and we get $\left[M_{1}\right]=\left[M_{2}\right]$, that is, $M_{1} \oplus L_{2} \simeq K \simeq M_{2} \oplus L_{1}$.

Definition. A $\Lambda$-module $M$ is said to be of almost type $K$ if $M \otimes_{\Lambda} Z$ is finite or equivalently if $\operatorname{rank}_{Z}\left(M \otimes_{\Lambda} Z\right)=0$.

EXAMPLES. Type $K$ modules and pseudo null modules are almost type $K$, $Z_{2}=\Lambda /(2(t-1))$ is almost type $K$ but not type $K$.

PROPOSITION (1.23). If $M$ is a $\Lambda$-torsion-free module, then $\operatorname{rank}_{Z}\left(M \otimes_{\Lambda} Z\right)=$ $\operatorname{rank}_{\Lambda}(M)$.

If $N$ is pseudo null, the long sequence of $\operatorname{Tor}_{\Lambda}$ derived from $0 \rightarrow \Lambda \stackrel{(t-1)}{\rightarrow} \Lambda \rightarrow$ $Z \rightarrow 0$ gives us,

$$
0=\operatorname{Tor}_{\Lambda}^{1}(N, \Lambda) \rightarrow \operatorname{Tor}_{\Lambda}^{1}(N, Z) \rightarrow N \stackrel{(t-1)}{\rightarrow} N \rightarrow N \otimes_{\Lambda} Z \rightarrow 0
$$

so $\operatorname{Tor}_{\Lambda}^{1}(N, Z)=\operatorname{kernel}[(t-1) N \rightarrow N]$ which is finite.

Consider now $0 \rightarrow M \rightarrow M^{* *} \rightarrow N \rightarrow 0, N$ pseudo null.

We have $M^{* *}=\Lambda^{r}$ where $r=\operatorname{rank}_{\Lambda} M$.

We obtain

$$
0 \rightarrow \operatorname{Tor}_{\Lambda}^{1}(N, Z) \rightarrow M \otimes_{\Lambda} Z \stackrel{\phi}{\rightarrow} Z^{r} \rightarrow N \otimes_{\Lambda} Z \rightarrow 0
$$

where both $N \otimes_{\Lambda} Z$ and $\operatorname{Tor}_{\Lambda}^{1}(N, Z)$ are finite so $\operatorname{rank}_{Z}\left(M \otimes_{\Lambda} Z\right)=r=\operatorname{rank}_{\Lambda}(M)$.

Notice that

$$
0 \rightarrow \operatorname{Tor}_{\Lambda}^{1}(N, Z) \rightarrow M \otimes_{\Lambda} Z \rightarrow \text { image } \phi \rightarrow 0
$$

is exact and image $\phi \simeq Z^{r}$, so

$$
M \otimes_{\Lambda} Z \simeq_{Z} Z^{r} \oplus \operatorname{Tor}_{\Lambda}^{1}(N, Z),
$$

that is, the $Z$-torsion part of $M \otimes_{\Lambda} Z$ is $\operatorname{Tor}_{\Lambda}^{1}(N, Z)$ when $M$ is $\Lambda$-torsion free. 
If $M$ is not $\Lambda$-torsion free we have

$$
\operatorname{rank}_{\Lambda}(M)=\operatorname{rank}_{\Lambda}(F T(M))=\operatorname{rank}_{Z}\left(F T(N) \otimes_{\Lambda} Z\right) .
$$

PROPOSITION (1.24). For free or torsion free $\Lambda$-module of rank one, the stable class determines the isomorphism class and conversely.

For free modules the proof is obvious, for ideals see [V.1].

I do not know if the proposition is true for modules of rank $\geq 2$.

Proposition (1.25). Let $0 \rightarrow M_{1} \stackrel{\phi}{\rightarrow} M_{2} \rightarrow Z^{r} \rightarrow 0$ be exact with $M_{2} \otimes_{\Lambda} Z \simeq$ $Z^{r}$. Then the submodule $\phi\left(M_{1}\right)=(t-1) M_{2}$.

Notice that $\operatorname{rank}_{\Lambda} M_{1}=\operatorname{rank}_{\Lambda} M_{2}$ and $(t-1)\left(M_{2} / \phi\left(M_{1}\right)\right)=(t-1) Z^{r}=0$ so $(t-1) M_{2} \subset \phi\left(M_{1}\right)$.

We also have the exact sequence

$$
0 \rightarrow \phi\left(M_{1}\right) /(t-1) M_{2} \rightarrow M_{2} /(t-1) M_{2} \rightarrow M_{2} / \phi\left(M_{1}\right) \rightarrow 0
$$

which is

$$
0 \rightarrow \phi\left(M_{1}\right) /(t-1) M_{2} \rightarrow Z^{r} \rightarrow Z^{r} \rightarrow 0
$$

and so $\phi\left(M_{1}\right)=(t-1) M_{2}$.

PROPOSITION (1.26). If $T$ is a $\Lambda$-torsion and pseudo null free module and there exists a Hermitian pairing $\langle\rangle:, T \times \bar{T} \rightarrow Q(\Lambda) / \Lambda$ with null space $K$ and conull space $C$, then $K \simeq e^{1}(\bar{C})$. If we let $T^{1}=T / K$, the induced pairing $\langle,\rangle^{1}: T^{1} \times$ $\bar{T}^{1} \rightarrow Q(\Lambda) / \Lambda$ is nondegenerate with conull space isomorphic to $e^{2}(\bar{C})$ and the homological dimension of $T^{1} \leq 1$ (clearly homological $\operatorname{dim} T \leq 1$ by 1.18).

For the proof see [L.2].

2. Characterization. This section is devoted to finding properties and relations for the family of $\Lambda$-modules $\left\{H_{i}(\hat{X}(\ell))\right\}$ and selecting those which are suitable for its characterization.

We are mainly concerned with links of dimension bigger than one, but some results on the classical case are presented.

$X(\ell)$ (or just $X$ ) will denote the complement of a open tubular neighborhood of a link $\ell: \bigcup_{i=1}^{m} K_{i}^{n} \rightarrow S^{n+2}$ and so it is a $(n+2)$-dimensional, oriented, compact connected manifold and its boundary is the disjoint union of $m$-copies of manifolds homeomorphic to $S^{n} \times S^{1}$.

Using duality we find that $H_{0}(X)=Z, H_{1}(X) \simeq Z^{m}, H_{n+1}(X) \simeq Z^{m-1}$ and $H_{i}(X)=0$ for $i \neq 0,1,(n+1)$ and so it depends only on the multiplicity $(m)$ and the dimension $(n)$ of the link.

The group of the link, $\pi_{1}(X)$, has some properties which, in many cases, are sufficient for its characterization, see $[\mathbf{K}]$.

The covering we are studying, $\hat{X}$, is defined by a surjection $\alpha: \pi_{1}(X) \rightarrow Z$ and we denote the kernel of $\alpha$, which is $\pi_{1}(\hat{X})$, by $\hat{\pi}$.

The $\Lambda$-structure of $H_{1}(\hat{X})$ can be defined in a purely algebraic way as follows.

The commutator $[\hat{\pi}, \hat{\pi}]$ is normal in $\pi$ and so we have a exact sequence $1 \rightarrow$ $\hat{\pi} /[\hat{\pi}, \hat{\pi}] \rightarrow \pi /[\hat{\pi}, \hat{\pi}] \rightarrow \pi / \hat{\pi} \rightarrow 0$.

The last group is $Z$ and we choose for it a generator $\alpha$. 
Conjugation in $\hat{\pi} /[\hat{\pi}, \hat{\pi}]$ by a pull back of $\alpha$ in $\pi /[\hat{\pi}, \hat{\pi}]$ is independent of the choice of the pull back.

This $Z$-action on $\hat{\pi} /[\hat{\pi}, \hat{\pi}]=H_{1}(\pi) \simeq H_{1}(\hat{X})$ defines a $\Lambda$-module structure on $H_{1}(\hat{X})$. The right choice of $\alpha$ makes this $\Lambda$-structure identical of that defined on $H_{1}(\hat{X})$ by covering transformations.

For more details see [L.1 and $\mathbf{N}]$.

There is no loss if we study coverings of the space $X$ instead of the complement of the link itself since there are retractions from this one to $X$ which can be lifted to the coverings.

The total linking number covering can be described geometrically with the help of a (not necessarily connected) Seifert manifold for the link. The Seifert manifold always exists, see [L.3].

The construction of $\hat{X}$ is the same as the construction of the universal abelian covering of knot complements, namely, by gluing conveniently spaces $X_{i}, i \in Z$, where each $X_{i}$ is a copy of $X(\ell)$ opened along the Seifert manifold.

Notice that $\partial \hat{X}$ is a disjoint union of manifolds homeomorphic to $S^{n} \times R$ and that the Seifert manifold mentioned in $X(\ell)$ is what remains of a Seifert manifold of the link in $S^{n+2}$ once we remove a convenient tubular neighborhood of the link.

The Cartan-Leray spectral sequence of the coverings $\hat{X} \rightarrow X$ degenerates and we obtain exact sequences

$$
\begin{aligned}
& \cdots 0 \rightarrow H_{n-1}(\hat{X}) \rightarrow \cdots \rightarrow H_{i}(\hat{X}) \stackrel{(t-1)}{\rightarrow} H_{i}(\hat{X}) \\
& \quad \rightarrow H_{i}(X) \rightarrow \cdots \rightarrow H_{0}(X) \rightarrow 0 \\
& \begin{aligned}
\cdots 0 \rightarrow H_{n+2}(X, \partial X) \rightarrow \cdots \rightarrow H_{i}(\hat{X}, \partial \hat{X}) \stackrel{(t-1)}{\rightarrow} H_{i}(\hat{X}, \partial \hat{X}) \\
\quad \rightarrow H_{i}(X, \partial X) \rightarrow \cdots \rightarrow H_{1}(X, \partial X) \rightarrow 0
\end{aligned}
\end{aligned}
$$

From now on, in this section, we use the notation $B_{i}=H_{i}(\hat{X})$ and $C_{i}=$ $H_{i}(\hat{X}, \partial \hat{X}), n$ is the dimension and $m$ the multiplicity of $\ell: \bigcup_{i=1}^{m} K_{i}^{n} \rightarrow S^{n+2}$.

PROPOSITION (2.1). $B_{q}$ and $C_{q}$ are finitely generated $\Lambda$-modules.

The proof is the same as Proposition (1.1) in [L.1].

Proposition (2.2). For links of dimension $n \geq 2$, the $\Lambda$-modules $B_{i}, i=$ $2,3, \ldots, n$, are of type $K$.

For $i=2,3, \ldots,(n-1)$ the conclusion comes from the exact sequence $(1)$ and the fact that $H_{j}(X)=0$ for $j=2,3, \ldots,(n-1)$.

For $i=n$ we observe that $H_{0}\left(Z ; H_{n}(\hat{X})\right)=0$ and from the Cartan-Leray spectral sequence we get $(t-1): B_{n} \rightarrow B_{n}$ surjective. But $\Lambda$ is noetherian so $(t-1): B_{n} \rightarrow$ $B_{n}$ is an isomorphism, that is, $B_{n}$ is of type $K$.

PROPOSITION (2.3). For links of dimension $n \geq 2$, we have exact sequences

$$
\begin{aligned}
& 0 \rightarrow B_{1} \rightarrow C_{1} \rightarrow Z^{m-1} \rightarrow 0, \\
& 0 \rightarrow B_{n+1} \rightarrow C_{n+1} \rightarrow Z^{m-1} \rightarrow 0
\end{aligned}
$$

and for $i=2,3, \ldots, n$ we have $B_{i} \simeq C_{i}$.

Following the steps of Lemma $(2.5)$ of $[\mathbf{L . 1}]$, we get $\partial: H_{n+1}(\hat{X}, \partial \hat{X}) \rightarrow H_{n}(\partial \hat{X})$ surjective so we obtain the exact sequences and the isomorphisms directly from the 
long exact sequence of the pair $(\hat{X}, \partial \hat{X})$ using the knowledge that $\partial \hat{X}$ is homeomorphic to $S^{n} \times R$.

Corollary (2.4). The modules $C_{i}, i=2,3, \ldots, n$, are of type $K$.

COROllary (2.5). The modules $B_{i}$ and $C_{i}, i=2,3, \ldots, n$, are $\Lambda$-torsion (see Corollary (13) of [L.1] for the proof).

Proposition (2.6). $B_{1} \otimes Z \simeq Z^{m-1} \simeq C_{1} \otimes Z$ and for $n \geq 2 B_{n+1} \otimes Z \simeq$ $Z^{m} \simeq C_{n+1} \otimes Z$.

We have by Proposition (1.4)(h) that $M \otimes Z \simeq$ cokernel of $\{(t-1): M \rightarrow M\}$ and so the results come from the sequences (1) and (2) using the fact that $B_{n}$ and $C_{n}$ are of type $K$ if $n \geq 2$.

Proposition (2.7). Let $H_{e}^{*}(\hat{X})$ and $H_{e}^{*}(\hat{X}, \partial \hat{X})$ denote the equivariant cohomology groups of $\hat{X}$ and $(\hat{X}, \partial \hat{X})$, respectively. Then there are duality isomorphisms $H_{n+2-i}(\hat{X}) \simeq \overline{H_{e}^{i}(\hat{X}, \partial \hat{X})}$ and $H_{i}(\hat{X}, \partial \hat{X}) \simeq \overline{H_{e}^{n+2-i}(\hat{X})}$ for $i=0,1, \ldots,(n+2)$.

For the proof and more details on the $\Lambda$-structure of these groups, see $\S 5$ of [L.1].

Proposition (2.8). For links of dimension $n \geq 2$ :
(a) $\overline{C_{n+1}} \simeq B_{1}^{*} \otimes Z$,
(a') $\overline{B_{n+1}} \simeq C_{1}^{*}$; the following sequences are exact:
(b) $0 \rightarrow e^{2}\left(B_{i-2}\right) \rightarrow \bar{C}_{n+2-i} \rightarrow e^{1}\left(B_{i-1}\right) \rightarrow 0,2 \leq i \leq n$,
$\left(\mathrm{b}^{\prime}\right) 0 \rightarrow e^{2}\left(C_{i-2}\right) \rightarrow \bar{B}_{n+2-i} \rightarrow e^{1}\left(C_{i-1}\right) \rightarrow 0,2 \leq i \leq n$,
(c) $0 \rightarrow F T\left(\bar{C}_{1}\right) \rightarrow B_{n+1}^{*} \rightarrow e^{2}\left(B_{n}\right) \rightarrow 0$,
$\left(\mathrm{c}^{\prime}\right) 0 \rightarrow F T\left(\bar{B}_{1}\right) \rightarrow C_{n+1}^{*} \rightarrow e^{2}\left(C_{n}\right) \rightarrow 0$,
(d) $0 \rightarrow e^{2}\left(B_{n-1}\right) \rightarrow T\left(\bar{C}_{1}\right) \rightarrow e^{1}\left(B_{n}\right) \rightarrow 0$,
$\left(\mathrm{d}^{\prime}\right) 0 \rightarrow e^{2}\left(C_{n-1}\right) \rightarrow T\left(\bar{B}_{1}\right) \rightarrow e^{1}\left(C_{n}\right) \rightarrow 0$.

Consider the universal coefficient spectral sequence for $C_{*}(\hat{X})$ which converges to the equivariant cohomology of $\hat{X}$.

$\Lambda$ has homological dimension 2 so $e^{i}\left(B_{p}\right)=0$ for $i \geq 3$.

$\hat{X}$ is a $(n+2)$-dimension manifold with boundary so $e^{q}\left(B_{j}\right)=0$ for $j \geq(n+2)$.

Since $B_{0} \simeq Z$ is $\Lambda$-torsion and pseudo null free, $e^{0}\left(B_{0}\right)=0=e^{2}\left(B_{0}\right)$.

By Proposition (2.7) we get $H_{e}^{i}(\hat{X})=0$ for $i \geq(n+2)$ and so $e^{2}\left(B_{n+1}\right)=0=$ $e^{1}\left(B_{n+1}\right)$.

$B_{i}$ is $\Lambda$-torsion for $i=2,3, \ldots, n$ and so, for $2 \leq i \leq n, e^{0}\left(B_{i}\right)=B_{i}^{*}=0$.

The $E^{3}$ term of the spectral sequence is $E^{\infty}$ which gives exact sequences:

$$
\begin{gathered}
0 \rightarrow e^{1}\left(B_{0}\right) \rightarrow H_{e}^{1}(X) \rightarrow B_{1}^{*} \rightarrow 0, \\
0 \rightarrow e^{2}\left(B_{i-2}\right) \rightarrow H_{e}^{i}(X) \rightarrow e^{1}\left(C_{i-1}\right) \rightarrow 0, \\
0 \rightarrow T\left(H_{e}^{n+1}(X)\right) \rightarrow H_{e}^{n+1}(X) \rightarrow B_{n+1}^{*} \rightarrow e^{2}\left(B_{n}\right) \rightarrow 0, \\
0 \rightarrow e^{2}\left(B_{n+1}\right) \rightarrow T\left(H_{e}^{n+1}(X)\right) \rightarrow e^{1}\left(B_{n}\right) \rightarrow 0 .
\end{gathered}
$$

(a), (b), (c), and (d) follow now by Propositions (2.7), (1.15), (1.10) and (1.4)(j); the other cases follow similarly from the Universal Coefficient Spectral Sequence for $C_{*}(\hat{X}, \partial \hat{X})$.

Notice that in this spectral sequence we have $H_{e}^{i}(\hat{X}, \partial \hat{X})=0$ for $i \geq(n+3)$ only, but on the other hand the differential $d: C_{n+1}^{*} \rightarrow e^{2}\left(C_{n}\right)$ must be surjective otherwise. 
$0 \neq$ cokernel $d \subset H^{n+2}(\hat{X}, \partial \hat{X}) \simeq Z$ which is impossible because $Z$ is pseudo null free and cokernel of $d$ is pseudo null.

Proposition (2.9). For classical lirks $(n=1)$, we have

(a) $\bar{C}_{2} \simeq Z \otimes B_{1}^{*}$

(a') $\bar{B}_{2} \simeq C_{1}^{*}$,

(b) $T\left(\bar{C}_{1}\right) \simeq e^{1}\left(B_{1}\right)$,

(b') $T\left(\bar{B}_{1}\right) \simeq e^{1}\left(C_{1}\right)$,

(c) $e^{1}\left(B_{2}\right)=0=e^{2}\left(B_{2}\right)$,

(c') $e^{1}\left(C_{2}\right) \simeq Z \simeq \bar{B}_{0}$ and $e^{2}\left(C_{2}\right)=0$,

(d) $0 \rightarrow e^{1}\left(B_{1}\right) \rightarrow \bar{C}_{1} \rightarrow B_{2}^{*} \rightarrow e^{2}\left(B_{1}\right) \rightarrow 0$ is exact,

$\left(\mathrm{d}^{\prime}\right) 0 \rightarrow e^{1}\left(C_{1}\right) \rightarrow \bar{B}_{1} \rightarrow C_{2}^{*} \rightarrow e^{2}\left(C_{1}\right) \rightarrow 0$ is exact.

The proof is similar to that of Proposition (2.8).

We observe now that from the last two propositions we can derive many results. For example, if $n \geq 2$,

$$
N\left(\bar{C}_{1}\right) \simeq e^{2}\left(B_{n+1}\right) \text { and } N\left(\bar{B}_{1}\right) \simeq e_{2}\left(C_{n-1}\right) .
$$

We consider now the inclusion map $j: \hat{X} \rightarrow(\hat{X}, \partial \hat{X})$ which induces the isomorphisms $B_{i} \simeq C_{i}$ for $2 \simeq i \simeq n$ and $e^{q}\left(j_{i}\right): e^{q}\left(C_{i}\right) \simeq e^{q}\left(B_{i}\right)$ for $q \geq 0$.

From the exact sequence $0 \rightarrow B_{1} \rightarrow C_{1} \rightarrow Z^{m-1} \rightarrow 0$, we obtain the exact sequence

$$
0 \rightarrow C_{1}^{*} \rightarrow B_{1}^{*} \rightarrow Z^{m-1} \rightarrow e^{1}\left(C_{1}\right) \rightarrow e^{1}\left(B_{1}\right) \rightarrow 0 .
$$

$\left(e^{2}\left(Z^{m-1}\right)\right)=0$ by Proposition $(1.4)(\mathrm{c})$.

Now we observe that $e^{1}\left(C_{1}\right) \simeq \bar{B}_{n} \simeq \bar{C}_{n} \simeq e^{1}\left(B_{1}\right)$. Thus, $e^{1}\left(j_{1}\right)$ is an epimorphism between isomorphic modules over a Noetherian ring $\Lambda$ and so is an isomorphism.

We have then $0 \rightarrow C_{1}^{*} \rightarrow B_{1}^{*} \rightarrow Z^{m-1} \rightarrow 0$ exact and $e^{1}\left(j_{1}\right): e^{1}\left(C_{1}\right) \stackrel{\approx}{\rightarrow} e^{1}\left(B_{1}\right)$ and $e^{2}\left(j_{1}\right): e^{2}\left(C_{1}\right) \stackrel{\approx}{\rightarrow} e^{2}\left(B_{1}\right)$. This exact sequence tensored with $Q(\Lambda)$ shows that $\operatorname{rank}_{\Lambda} C_{2}=\operatorname{rank}_{\Lambda} B_{1}$.

For the classical case we have the exact sequence

$$
0 \rightarrow B_{2} \stackrel{(t-1)}{\rightarrow} B_{2} \stackrel{\phi}{\rightarrow} Z^{m} \rightarrow B_{1} \rightarrow B_{1} \rightarrow Z^{m-1} \rightarrow 0 .
$$

Setting $r(\ell)=\operatorname{rank}_{Z}$ (image $\left.\phi\right)$, we have $r \leq(m-1)$ and $0 \rightarrow B_{2} \rightarrow B_{2} \rightarrow$ $Z^{r} \rightarrow 0$ exact. Since $B_{2}$ is $\Lambda$-free by Proposition (2.9) we have rank $B_{2}=r(\ell)$.

PROPOSITION (2.10). For a link $\ell$ of dimension $n$ and multiplicity $m$ we have

(a) if $n \geq 2$, rank $B_{1}=\operatorname{rank} C_{1}=\operatorname{rank} B_{n+1}=\operatorname{rank} C_{n+1}=(m-1)$,

(b) if $n=1$, rank $B_{1}=\operatorname{rank} C_{1}=\operatorname{rank} B_{2}=\operatorname{rank} C_{2}=r(\ell) \leq(m-1)$.

The proof follows by the above observations, the isomorphisms of Propositions (2.8) and (2.9), and the exact sequence $0 \rightarrow B_{n+1} \stackrel{(t-1)}{\rightarrow} B_{n+1} \rightarrow Z^{m-1} \rightarrow 0$.

In the classical case, $B_{2} \simeq \Lambda^{r}$ and $C_{2} \simeq \Lambda^{r} \oplus Z$, so the rank $B_{1}(=r)$ does not determine the multiplicity.

The triple $\left(B_{1}(\ell), C_{1}(\ell), m\right)$ associated to a link $\ell$ is in this case the right choice of invariants.

We collect in the next proposition the results on the classical case. 
THEOREM (2.11). For a link $\ell: \bigcup_{i=1}^{m} S_{i}^{1} \rightarrow S^{3}$ there is associated a triple $\left(B_{1}, C_{1}, m\right) ; B_{1}=H_{1}(\hat{X}(\ell)) ; C_{1}=H_{1}(\hat{X}(\ell), \partial \hat{X}(\ell))$ and $m$ the multiplicity, such that

(a) rank $B_{1}=\operatorname{rank} C_{1}=r \leq(m-1)$,

(b) there is a exact sequence of $\Lambda$-modules

$$
0 \rightarrow \Lambda^{r} \rightarrow \Lambda^{r} \oplus Z \rightarrow Z^{m} \rightarrow B_{1} \rightarrow C_{1} \rightarrow Z^{m-1} \rightarrow 0
$$

(c) $T\left(\bar{C}_{1}\right)=e^{1}\left(B_{1}\right)$ and $T\left(\bar{B}_{1}\right) \simeq e^{1}\left(C_{1}\right)$.

The stable classes $\left[F T\left(\bar{B}_{1}\right)\right]$ and $\left[F T\left(\bar{C}_{1}\right)\right]$ determine and are determined by the isomorphism classes $\left\{e^{2}\left(C_{1}\right)\right\}$ and $\left\{e^{2}\left(B_{1}\right)\right\}$, respectively.

From now on, we restrict our attention to the case $n \geq 2$.

PROPOSITION (2.12). For a link of dimension $n \geq 2, T\left(C_{1}\right)$ and $T\left(B_{1}\right)$ are of type $K$.

For $n>2$, use Proposition (2.8) and the fact that $C_{n}, C_{n-1}, B_{n}, B_{n-1}$ are of type $K$.

For $n=2$, we have $B_{2}$ of type $K$ (Proposition (2.2)), and so $e^{2}\left(B_{2}\right)$ is of type $K$. It follows that $\operatorname{Tor}_{\Lambda}^{i}\left(e^{2}\left(B_{2}\right) ; Z\right)=0=\operatorname{Ext}_{\Lambda}^{i}\left(e^{2}\left(B_{2}\right) ; Z\right)$ for all $i$ (Proposition (1.4)(i)).

From the exact sequence $0 \rightarrow F T\left(\bar{B}_{1}\right) \rightarrow C_{3}^{*} \rightarrow e^{2}\left(B_{2}\right) \rightarrow 0$, we get

$$
0=\operatorname{Tor}_{\Lambda}^{i}\left(e^{2}\left(B_{2}\right) ; Z\right) \rightarrow F T\left(\bar{B}_{1}\right) \otimes_{\Lambda} Z \rightarrow C_{3}^{*} \otimes Z \rightarrow e^{2}\left(B_{2}\right) \otimes_{\Lambda} Z=0
$$

and so $Z^{m-1} \simeq C_{3}^{*} \otimes Z \simeq F T\left(\bar{B}_{1}\right) \otimes Z$.

From the exact sequence $0 \rightarrow T\left(B_{1}\right) \rightarrow B_{1} \rightarrow F T\left(B_{1}\right) \rightarrow 0$ we get the exact sequence

$$
0=\operatorname{Tor}_{\Lambda}^{1}\left(F T\left(B_{1}\right), Z\right) \rightarrow T\left(B_{1}\right) \otimes Z \rightarrow B_{1} \otimes Z \rightarrow F T\left(B_{1}\right) \otimes Z \rightarrow 0,
$$

or $0 \rightarrow T\left(B_{1}\right) \otimes Z \rightarrow Z^{m-1} \rightarrow Z^{m-1} \rightarrow 0$, and so $T\left(B_{1}\right) \otimes Z=0$. Hence, $T\left(B_{1}\right)$ is of type $K$.

Similarly, $T\left(C_{1}\right)$ is of type $K$.

PROPOSITION (2.13). For a link of dimension $n \geq 2$, the map, $j: \hat{X} \rightarrow$ $(\hat{X}, \partial \hat{X})$ induces an isomorphism $T\left(j_{1}\right): T\left(B_{1}\right) \rightarrow T\left(C_{1}\right)$ and the submodule $(t-$ 1). $C_{1} \subset C_{1}$ is $j_{1}\left(B_{1}\right)$.

From the exact sequence $0 \rightarrow B_{1} \stackrel{j_{1}}{\rightarrow} C_{1} \rightarrow Z^{m-1} \rightarrow 0$ and Propositions (2.6) and (1.25), we get $j_{1}\left(B_{1}\right)=(t-1) C_{1}$. Observe that $(t-1): C_{1} \rightarrow C_{1}$ is injective because $H_{2}(X, \partial X)=0$. Since $T\left(C_{1}\right)$ is of type $K,(t-1): T\left(C_{1}\right) \simeq T\left(C_{1}\right)$ is of type $K$ and so $j_{1}\left(B_{1}\right)=T\left(C_{1}\right)$.

PROPOSITION (2.14). For a link of dimension $n \geq 2$, there is a commutative diagram:

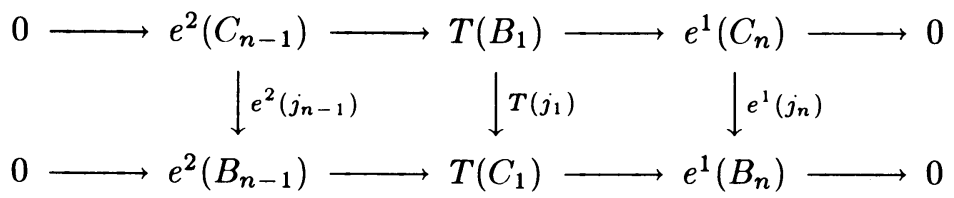


Consider the map $j_{*}: C_{*}(\hat{X}) \rightarrow C_{*}(\hat{X}, \partial \hat{X})$ which induces a map between the universal coefficient spectral sequences of $C_{*}(\hat{X})$ and $C_{*}(\hat{X}, \partial \hat{X})$.

We denote by $X^{1}$ and $X^{2}$ the first and second dual complexes, respectively, of a cellular decomposition of $X$.

We observe that $X$ embeds in $X^{2}\left(X \stackrel{\lambda}{\rightarrow} X^{2}\right)$ and that there is a cellular retraction $r: X^{2} \rightarrow X\left(r \circ \lambda=\operatorname{Id}_{x}\right)$.

Let $\hat{\lambda}$ be the lifting $\lambda$ to $\hat{X}$. Then there is a duality homomorphism $\psi_{\lambda}: C_{q}(\hat{X}) \rightarrow$ $\left(C_{n+2-q}\left(\hat{X}^{1}, \partial \hat{X}^{1}\right)\right)^{*}$ given by composing $\lambda$ with the usual duality $\psi: C_{q}\left(\hat{X}^{2}\right) \rightarrow$ $\left(C_{n+2-q}\left(\hat{X}^{1}, \partial \hat{X}^{1}\right)\right)^{*}$.

This yields a commutative diagram

$$
\begin{array}{ccc}
H_{e}^{n+1}\left(X^{1}, \partial X^{1}\right) & \stackrel{\psi_{\lambda}}{\approx} & H_{1}(\hat{X}) \\
\downarrow H_{e}^{n+1}\left(j_{n+1}^{1}\right) & & \downarrow H_{1}\left(j_{1}\right) \\
H_{e}^{n+1}\left(X^{1}\right) & \stackrel{\phi}{\longleftarrow} & H_{1}(\hat{X}, \partial \hat{X})
\end{array}
$$

where $\phi$ and $\psi_{\lambda}$ are duality isomorphisms.

The map $j_{*}: C_{*}(\hat{X}) \rightarrow C_{*}(\hat{X}, \partial \hat{X})$ gives a commutative diagram

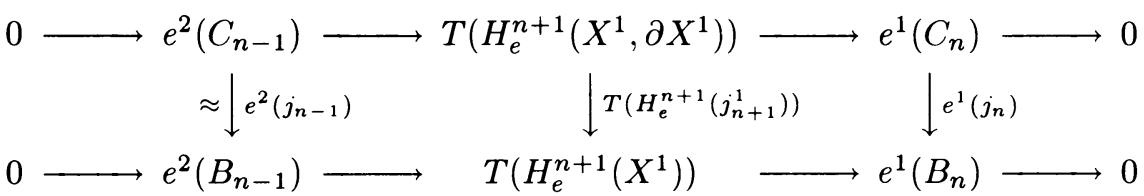

coming from the induced map on the spectral sequences.

The result follows by composing now the second diagram with the torsion part of the first diagram.

Observe that for $n \geq 2, B_{i} \simeq C_{i}$ if $2 \leq i \leq n, B_{n+1} \simeq \Lambda^{m-1}, C_{n+1} \simeq \Lambda^{m-1} \oplus Z$, $B_{1} \simeq(t-1) C_{1} \simeq C_{1}$ and $\left.T\left(B_{1}\right) \simeq T\left(C_{1}\right)\right)$.

The rank of $B_{1}$ determines the multiplicity of the link.

The $n$-tuple $\left(B_{i}\right)_{i=1,2, \ldots, n}$ is then our choice of invariants.

PROPOSITION (2.15). For a link of dimension $n \geq 2$ there are pairings

$\langle\rangle:, F N\left(B_{q}\right) \times F N\left(B_{n+1-q}\right) \rightarrow Q(\Lambda) / \Lambda, 2 \leq q \leq(n-1)$,

$\langle\rangle:, F N\left(T\left(B_{1}\right)\right) \times F N\left(B_{n}\right) \rightarrow Q(\Lambda) / \Lambda$, and

$[]:, N\left(B_{q}\right) \times N\left(B_{n-q}\right) \rightarrow Q / Z, 1 \leq q \leq n-1$, such that

$\langle$,$\rangle is conjugate linear and nonsingular,$

[, ] is $Z$ linear, conjugate selfadjoint and nonsingular.

The first pairings are called Blanchfield pairings and the second ones are called Levine pairings.

For $2 \leq q \leq(n-1)$ we have

$$
\begin{aligned}
F N\left(B_{q}\right) & \simeq e^{1}\left(\bar{C}_{n+1-q}\right) \quad(\text { Proposition } 2.8) . \\
e^{1}\left(C_{n+1-q}\right) & \simeq e^{1}\left(B_{n+1-q}\right) \quad(\text { Proposition } 2.1) . \\
e^{1}\left(B_{n+1-q}\right) & \simeq e^{1}\left(F N\left(B_{n+1-q}\right)\right) \\
& \simeq \operatorname{Hom}_{\Lambda}\left(F N\left(B_{n+1-q}\right) ; Q(\Lambda) / \Lambda\right) \quad(\text { Proposition 1.4). }
\end{aligned}
$$


Similarly, $F N\left(T\left(B_{1}\right)\right) \simeq e^{1}\left(\bar{C}_{n}\right) \simeq e^{1}\left(\bar{B}_{n}\right) \simeq \operatorname{Hom}_{\Lambda}\left(\bar{B}_{n}, Q(\Lambda) / \Lambda\right)$, so we can define the pairing $\langle$,$\rangle by the composition of these isomorphisms. The above$ properties follow directly from the definitions.

For $1 \leq q \leq(n-1)$ we have by Proposition $2.8, N\left(B_{q}\right) \simeq e^{2}\left(\bar{C}_{n-q}\right)$; by Proposition 2.1, $e^{2}\left(\bar{C}_{n-q}\right) \simeq e^{2}\left(\bar{B}_{n-q}\right)$ and by Proposition $1.16, e^{2}\left(\bar{B}_{n-q}\right) \simeq$ $\operatorname{Hom}_{Z}\left(N\left(B_{n-q}\right), Q / Z\right)$. So, the pairing [, ] can be defined using this sequence of isomorphisms, and the properties follow.

Note that for $n=2 q-1$, the pairing $\langle\rangle:, F N\left(B_{q}\right) \times F N\left(B_{q}\right) \rightarrow Q(\Lambda) / \Lambda$ and, for $n=2 q$, the pairing [,] $: N\left(B_{q}\right) \times N\left(B_{q}\right) \rightarrow Q / Z$ have nice geometric interpretations which allow us to get some extra properties for them.

PROPOSITION (2.16). If $n=2 q-1, n \geq 3$, the Blanchfield pairing $\langle$,$\rangle :$ $F N\left(B_{q}\right) \times F N\left(B_{q}\right) \rightarrow Q(\Lambda) / \Lambda$ is conjugate linear, nonsingular and $(-1)^{q+1}$ Hermitian.

PROPOSITION (2.17). If $n=2 q, n \geq 4$, the Levine pairing [, ]: $N\left(B_{q}\right) \times$ $N\left(B_{q}\right) \rightarrow Q / Z$ is $Z$-linear, conjugate selfadjoint, $(-1)^{q+1}$-symmetric and nonsingular.

The proofs of these propositions follow exactly the same steps of $\S \S 5$ and 6 of [L.1]; one should only observe that in this range of indices all $\Lambda$-modules used in the arguments are of type $K$ and so pseudo null means $Z$-torsion and pseudo null free means $Z$-torsion free (see note after 1.19).

Proposition (2.18). The Levine pairing [, ] : $N\left(B_{1}\right) \times N\left(B_{1}\right) \rightarrow Q / Z$ of a link $\ell: \bigcup_{i=1}^{m} S_{i}^{2} \rightarrow S^{4}$ is a $Z$-linear, conjugate selfadjoint, symmetric nonsingular pairing.

The proof still follows from [L.1] with some adjustment.

We next define a preliminary pairing $\{,\}_{k}$, choosing $k$ large enough to satisfy certain conditions to be described during the discussion below.

Set $\theta_{k}=\Lambda\left(t^{k}-1\right)$ and let $I\left(\theta_{k}\right)$ be the injective hull of $\theta_{k}$. In the same way as in [L.1], it is enough to obtain a pairing $\{,\}_{k}: N\left(B_{1}\right) \times N\left(B_{1}\right) \rightarrow I\left(\theta_{k}\right) / \theta_{k}$ with the properties stated in the proposition. Construction of $\{,\}_{k}$ :

First of all, note that $N\left(B_{1}\right) \simeq N\left(H_{1}(\hat{X})\right) \stackrel{j_{1 *}^{*}}{\rightarrow} N\left(H_{1}(\hat{X}, \partial \hat{X})\right)$ and by duality the last $\Lambda$-module is isomorphic to $N\left(H_{e}^{3}(\hat{X})\right)$. Thus, we are looking for a homomorphism $N\left(H_{e}^{3}(\hat{X})\right) \rightarrow \operatorname{Hom}_{\Lambda}\left(N\left(B_{1}\right), I\left(\theta_{k}\right) / \theta_{k}\right)$.

Consider the chain of homomorphisms

$$
\begin{aligned}
& H_{e}^{3}(\hat{X}) \stackrel{\delta^{\prime}}{\leftarrow} H_{e}^{2}\left(\hat{X} ; \theta_{k}\right) \stackrel{\delta^{\prime \prime}}{\longleftarrow} H_{e}^{1}\left(\hat{X}, I\left(\theta_{k}\right) / \theta_{k}\right) \stackrel{e^{\prime}}{\rightarrow} \\
& \rightarrow \operatorname{Hom}_{\Lambda}\left(B_{1} ; I\left(\theta_{k}\right) / \theta_{k}\right) \stackrel{r}{\rightarrow} \operatorname{Hom}_{\Lambda}\left(N\left(B_{1}\right) ; I\left(\theta_{k}\right) / \theta_{k}\right)
\end{aligned}
$$

where $\delta^{\prime}$ and $\delta^{\prime \prime}$ are coboundary maps of the exact cohomology sequence derived from the short exact sequence $0 \rightarrow \Lambda \stackrel{t^{k}-1}{\rightarrow} \Lambda \rightarrow \theta_{k} \rightarrow 0$ and $0 \rightarrow \theta_{k} \rightarrow I\left(\theta_{k}\right) \rightarrow$ $I\left(\theta_{k}\right) / \theta_{k} \rightarrow 0 ; e^{\prime}$ is evaluation and $r$ is restriction.

Define $\phi_{k}: D_{k} \rightarrow \operatorname{Hom}_{\Lambda}\left(N\left(B_{1}\right) ; I\left(\theta_{k}\right) / \theta_{k}\right)$ by setting $\phi_{k}(\alpha)=r e^{\prime}\left(\alpha^{\prime}\right)$ if $\delta^{\prime} \cdot \delta^{\prime \prime}\left(\alpha^{\prime}\right)=\alpha$.

There is no change in the arguments of [L.1] to prove that $\phi_{k}$ is well defined.

The next step is to verify that $\phi_{k}$ is an isomorphism from

$$
N\left(H_{e}^{3}(\hat{X})\right) \rightarrow \operatorname{Hom}_{\Lambda}\left(N\left(B_{1}\right), I\left(\theta_{k}\right) / \theta_{k}\right) .
$$


Suppose $\alpha \in N\left(H_{e}^{3}(\hat{X})\right)$ and $\alpha^{\prime} \in H_{e}^{1}\left(\hat{X}, I\left(\theta_{k}\right) / \theta_{k}\right)$ are such that $\delta^{\prime} \delta^{\prime \prime}\left(\alpha^{\prime}\right)=\alpha$ and $r \cdot e^{\prime}\left(\alpha^{\prime}\right)=0$. We first want to prove injectivity of $\phi_{k}$, so we need to verify that $\alpha=0$.

For this, it is sufficient to verify that $\alpha^{\prime}$ lies in the submodule generated by the images of $H_{e}^{1}\left(X, I\left(\theta_{k}\right)\right)$ and $H_{e}^{1}(\hat{X}, Q(\Lambda) / \Lambda)$ under the appropriate coefficient homomorphism.

From the universal coefficient spectral sequence applied to $\Lambda$-modules $G$ of injective dimension one (like $I\left(\theta_{k}\right), I\left(\theta_{k}\right) / \theta_{k}$ and $Q(\Lambda) / \Lambda$ ), there are exact sequences $0 \rightarrow \operatorname{Ext}_{\Lambda}^{1}\left(H_{0}(\hat{X}) ; G\right) \rightarrow H_{e}^{1}(\hat{X}, G) \rightarrow \operatorname{Hom}_{\Lambda}\left(H_{1}(\hat{X}) ; G\right) \rightarrow 0$. Therfore there is a commutative diagram with exact rows

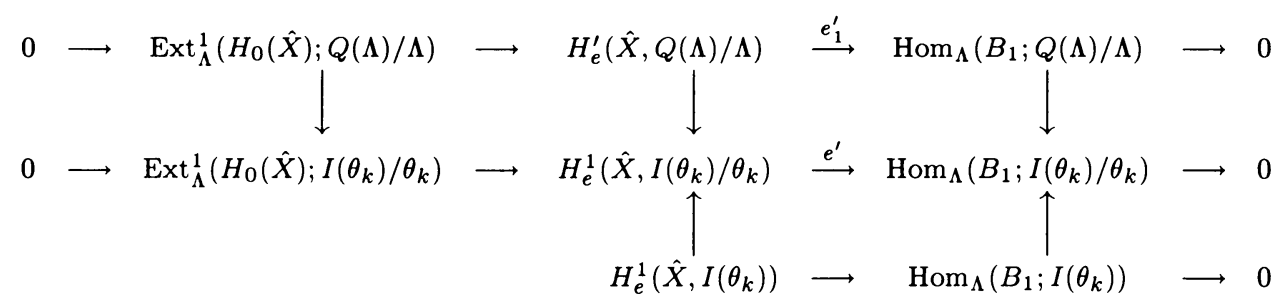

Furthermore, for any $\Lambda$-module $A$, we have a commutative diagram

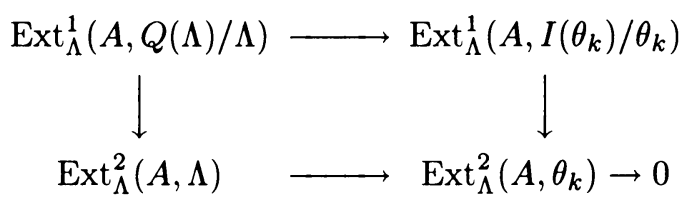

where the bottom row is a portion of a long exact sequence derived from the short exact sequence

$$
0 \rightarrow A \stackrel{t^{k}-1}{\rightarrow} \Lambda \rightarrow \theta_{k} \rightarrow 0 .
$$

Since the left vertical arrow in the first diagram is surjective, it is sufficient to show that $e^{\prime}\left(\alpha^{\prime}\right)$ lies in the submodule generated by the images of $\operatorname{Hom}_{\Lambda}\left(B_{1}, Q(\Lambda) / \Lambda\right)$ and $\operatorname{Hom}_{\Lambda}\left(B_{1}, I(\theta)\right)$.

We have that $r e^{\prime}\left(\alpha^{\prime}\right)=0$, that is, $e^{\prime}\left(\alpha^{\prime}\right)$ is induced by an element in

$$
\operatorname{Hom}_{\Lambda}\left(F N\left(B_{1}\right), I\left(\theta_{k}\right) / \theta_{k}\right),
$$

so the conclusion comes from the exactness of the following commutative diagram of exact rows and columns.

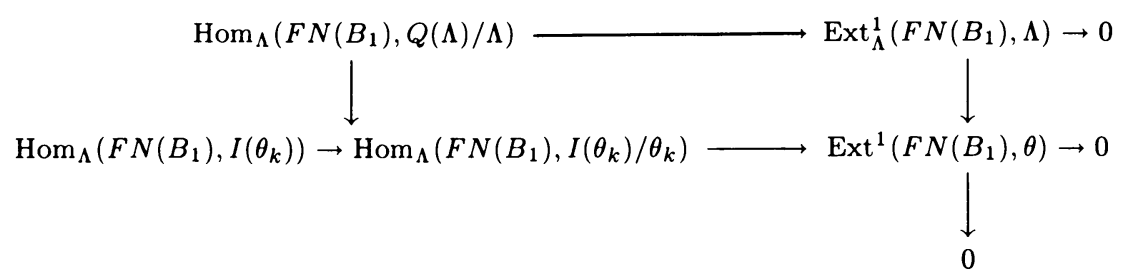

We prove now the surjectivity of $\phi_{k}$.

Observe that the universal coefficient spectral sequence give us that $e^{\prime}$ is surjective.

The restriction map $r$ is surjective because $e^{2}\left(F\left(N\left(B_{1}\right)\right)=0\right.$ (Proposition 1.4). Hence, $\operatorname{Ext}^{2}\left(F\left(N\left(B_{1}\right)\right), \theta\right)=0$ (use the long exact sequence of Ext derived for $\left.0 \rightarrow \Lambda \stackrel{t^{k}-1}{\rightarrow} \Lambda \rightarrow \theta \rightarrow 0\right)$. 
Furthermore, $\operatorname{Ext}_{\Lambda}^{2}\left(F N\left(B_{1}\right), \theta_{k}\right)=\operatorname{Ext}_{\Lambda}^{1}\left(F N\left(B_{1}\right), I\left(\theta_{k}\right) / \theta_{k}\right)=0$ (use the long exact sequence of Ext derived from $0 \rightarrow \theta_{k} \rightarrow I\left(\theta_{k}\right) \rightarrow I(Q) / \theta_{k} \rightarrow 0$ ), so the long exact sequence of Ext derived from $0 \rightarrow N\left(B_{1}\right) \rightarrow B_{1} \rightarrow F N\left(B_{1}\right) \rightarrow 0$ gives the surjectivity of $r$.

We next prove that we can choose $k$ so that $D_{k}=N\left(H_{e}^{3}(\hat{X})\right)$; the surjectivity of $\phi_{k}$ follows.

Let $m$ be an integer such that $m \cdot N\left(H_{e}^{3}(\hat{X})\right)=0$ and $\Lambda_{m}=\Lambda / m \Lambda$.

The equivariant cohomology group $H_{e}^{3}\left(\hat{X}, \Lambda_{m}\right)$ is a $\Lambda$-module and there is a commutative diagram with exact rows

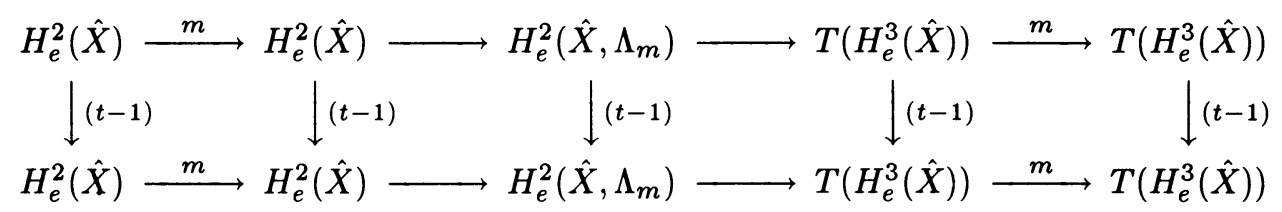

showing that $H_{e}^{2}\left(X ; \Lambda_{m}\right)$ is of type $K, Z$-torsion and so pseudo null and finite. Hence, we can find $k$ such that $\left(t^{k}-1\right)$ annihilates it.

For $\alpha \in N\left(H_{e}^{3}(\hat{X})\right)$, one can now follow the steps of $[\mathbf{L . 1}, \S 6]$ to obtain $\alpha^{\prime} \in$ $H_{e}^{1}\left(\hat{X}, I\left(\theta_{k}\right) / \theta_{k}\right)$ such that $\delta^{\prime} \delta^{\prime \prime}\left(\alpha^{\prime}\right)=\alpha$. Thus, we have found $k$ such that $D_{k}=$ $N\left(H_{e}^{3}(\hat{X})\right)$ which implies that $\phi_{k}: N\left(H_{e}^{3}(\hat{X})\right) \rightarrow \operatorname{Hom}_{\Lambda}\left(N\left(B_{1}\right), I\left(\theta_{k}\right) / \theta_{k}\right)$ is an isomorphism and Proposition (2.18) follows.

There is one more interesting characteristic of $F N\left(B_{2}(\ell)\right)$ for a link $\ell: \bigcup_{i=1}^{m} S_{i}^{3}$ $\rightarrow S^{5}$ which can be studied with the help of Seifert manifolds and Seifert matrices (see [L.1, $\S 14$ and 15]).

If $V$ is a Seifert manifold for a link and if $Y=S^{5}-V$, there are exact sequences:

$$
\begin{aligned}
& 0 \rightarrow t_{2}(V) \otimes_{Z} \Lambda \stackrel{d_{t}}{\rightarrow} t_{2}(Y) \otimes_{Z} \Lambda \rightarrow N\left(H_{2}(\hat{X})\right) \rightarrow 0, \\
& 0 \rightarrow f_{2}(V) \otimes_{Z} \Lambda \stackrel{d_{f}}{\rightarrow} f_{2}(Y) \otimes_{Z} \Lambda \rightarrow F N\left(H_{2}(\hat{X})\right) \rightarrow 0
\end{aligned}
$$

where $t_{2}(V)$ and $f_{2}(V)$ are the $Z$-torsion and $Z$-torsion free parts of $H_{2}(V)$.

We have a linking pairing $\ell: f_{2}(V) \times f_{2}(V) \rightarrow Z$ and we denote its matrix by $A$. There is a basis where $d_{f}$ is represented by $t A+A^{\prime}$ where $A^{\prime}$ is the transpose of $A$. One can compute the Blanchfield pairing on $F N\left(H_{2}(\hat{X})\right)$ with respect to a corresponding basis and get the matrix $(1-t)\left(t A+A^{\prime}\right)^{-1}$.

It is possible to verify that $A+A^{\prime}$ is a representative of the intersection pairing of $V$ and, since $\partial V$ is a union of spheres, the intersection pairing is unimodular and the signature of $A+A^{\prime}$ is a multiple of 16 (= index of $V$ ).

Using the function $x: Q(\Lambda) / \Lambda \rightarrow Q$ defined by Trotter in [T.1] we obtain from the Blanchfield pairing, a new pairing $\langle,\rangle_{x}: F N\left(B_{2}(\ell)\right) \otimes_{Z} Q \times F N\left(B_{2}(\ell)\right) \otimes_{Z}$ $Q \rightarrow Q$ and signature of $\langle,\rangle_{x}=$ index $V$.

PROPOSITION (2.19). Given a link $\ell: \bigcup_{i=1}^{m} S_{i}^{3} \rightarrow S^{5}$, the signature of $\langle,\rangle_{x}$ is a multiple of 16 .

For the proof see $[\mathbf{L . 1}, \S 15]$.

Collecting now the results we have obtained the following theorem.

THEOREM (2.20). Let $\ell: \bigcup_{i=1}^{m} K_{i}^{n} \rightarrow S^{n+2}$ be a link and $\alpha=\Sigma \cdot \Omega \cdot A$, $\alpha: \pi_{1}(X(\ell)) \rightarrow Z$ where $A$ is abelianization, $\Omega$ is isomorphism defined by choices of meridians and orientations, and $\Sigma$ is sum of coordinates of $Z^{m}$. 
Let $\hat{X}(\ell)$ be the covering associated to the kernel of $\alpha$ and $B_{i}=H_{i}(\hat{X}(\ell))$. Then

$$
\left(\pi_{1}(\hat{X}(\ell)), \alpha\right) \text { is an } m \text {-admissible pair, }
$$

(a) $\operatorname{rank}_{\Lambda} B_{1}=(m-1)$,

(b) $T\left(B_{1}\right), B_{2}, \ldots, B_{n}$ are of type $K$.

(c) $N\left(\bar{B}_{q}\right) \simeq \operatorname{Ext}_{\Lambda}^{2}\left(N\left(B_{n-q}\right), \Lambda\right)$ and $F N\left(\bar{B}_{q}\right) \simeq \operatorname{Ext}_{\Lambda}^{1}\left(F N\left(B_{n+1-q}\right), \Lambda\right)$ for $2 \leq$ $q \leq(n-1)$.

(d) $N\left(\bar{B}_{1}\right) \simeq \operatorname{Ext}_{\Lambda}^{2}\left(N\left(B_{n-1}\right), \Lambda\right)$ and $F N\left(T\left(\bar{B}_{1}\right)\right) \simeq \operatorname{Ext}_{\Lambda}^{1}\left(F N\left(B_{n}\right), \Lambda\right)$.

(e) $\bar{B}_{n} \simeq \operatorname{Ext}^{1}\left(F N\left(B_{1}\right), \Lambda\right)$.

(f) The stable class $\left[F T\left(\bar{B}_{1}\right)\right]$ determines and is determined by the isomorphism class of $\operatorname{Ext}_{\Lambda}^{2}\left(N\left(B_{n}\right), \Lambda\right)$.

(g) $B_{q} \otimes_{\Lambda} Z \simeq Z^{m-1}$.

(h) The Blanchfield pairing $\langle$, $\rangle: F N\left(T\left(B_{1}\right)\right) \times F N\left(T\left(B_{n+1-q}\right)\right) \rightarrow Q(\Lambda) / \Lambda$ is defined for $1 \leq q \leq(n+1)$. It is conjugate linear, nonsingular and, when $n=2 q-1$, it is $(-1)^{q+1}$-Hermitian.

(i) The Levine pairing [, ]: $N\left(B_{q}\right) \times N\left(B_{n-q}\right) \rightarrow Q / Z$ is defined for $1 \leq q \leq$ $(n-1)$. It is $Z$-linear, conjugate selfadjoint, nonsingular and, when $n=2 q$, it is symmetric.

(j) If $n=3$, the index of $\langle\rangle:, F N\left(T\left(B_{2}\right)\right) \otimes_{Z} Q \times F N\left(B_{2}\right) \otimes_{Z} Q \rightarrow Q$ is a multiple of 16 .

(k) $B_{1} \simeq H_{1}\left(\pi_{1}(\hat{X}(\ell))\right)$.

(l) The Hopf homomorphism $\rho: B_{2} \rightarrow H_{2}\left(\pi_{1}(\hat{X}(\ell))\right)$ is surjective.

3. Realization. Associated to a link $\ell: \bigcup_{i=1}^{m} K_{i}^{n} \rightarrow S^{n+2}$ we have the family of $\Lambda$-modules $\left\{H_{i}(\hat{X}(\ell))\right\}$ that have certain properties and are related in special ways.

In this section we study the possibility of constructing a link with a preassigned family of $\Lambda$-modules.

For knots this problem was solved in [L.1] with the exception of the pseudo null part of the first homology module. The same difficulty persists for links.

Many results of $[\mathbf{L . 1}$ and $\mathbf{K}]$ are used and are stated here in a convenient form.

We define a type of connected sum of links and develop some of its properties which are used in the realization work.

THEOREM (KERVAIRE) (3.1). A finitely presented group $G$ is the group of a link $\ell: \bigcup_{i=1}^{m} K_{i}^{n} \rightarrow S^{n+2}, n \geq 3$, if and only if

(i) $H_{1}(G) \simeq Z^{m}$,

(ii) weight $(G)=m$,

(iii) $H_{2}(G)=0$.

THEOREM (KERVAIRE) (3.2). If a group $G$ satisfies (i) and (ii) above and has a presentation of deficiency $m$, then there is a link $\ell: \bigcup_{i=1}^{m} S_{i}^{2} \rightarrow K^{4}$ where $K^{4}$ is a homotopy 4-sphere and $\pi_{1}(X(\ell))=G$.

ThEOREM (LEVINe) (3.3). Given a $\Lambda$-module $A$ of type $K$ and integers $q \geq 2, n \geq 2 q$ and with $A$ pseudo null free if $n=2 q$, then there is an $n$-knot $k$ (diffeomorphic to $S^{n}$ ) in $S^{n+2}$ such that

$\pi_{1}(X(k)) \simeq Z$, 


$$
\begin{aligned}
& H_{q}(\hat{X}(k)) \simeq A, \\
& H_{i}(\hat{X}(k))=0 \text { if } i \neq 0, q \text { and } 0<i \leq[n / 2] \text { and, } \\
& H_{i}(\hat{X}(k)) \text { pseudo null if } i=(n+1) / 2 .
\end{aligned}
$$

THEOREM (LEVINE) (3.4). Let $F$ be a pseudo null free $\Lambda$-module of type $K$ equipped with a pairing $\langle\rangle:, F \times F \rightarrow Q(\Lambda) / \Lambda$ which is conjugate linear, nonsingular and $(-1)^{q+1}$-Hermitian for some positive integer $q$.

If $n=2 q-1>3$, there exists an $n$-knot $k$ with $H_{q}(\hat{X}(k)) \simeq F$, Blanchfield pairing $\langle$,$\rangle and H_{i}(\hat{X}(k))=0$ for $i \neq 0, q$.

THEOREM (LEVINE) (3.5). Let $A$ be a pseudo null $\Lambda$-module of type $K$ and $[]:, A \times A \rightarrow Q / Z$ a $Z$-linear, $(-1)^{q+1}$-symmetric, nonsingular conjugate selfadjoint pairing. If $q>1$, there exists a $2 q$-knot $k$ having $H_{q}(\hat{X}(k)) \simeq A$, Levine pairing $[$,$] , and H_{i}(\hat{X}(k))=0$ for $i \neq 0, q$.

THEOREM (HOPF) (3.6). Given a link $\ell: \bigcup_{i=1}^{m} K_{i}^{n} \rightarrow S^{n+2}, n \geq 2$, there is a surjection $\rho: H_{2}(\hat{X}(\ell)) \rightarrow H_{2}\left(\pi_{1}(\hat{X}(\ell))\right)$.

This is just part of the Hopf sequence of $\hat{X}(\ell)$.

If we keep the notation of the last section, namely, $B_{i}(\ell)=H_{i}(\hat{X}(\ell)), \hat{\pi}(\ell)=$ $\pi_{1}(\hat{X}(\ell))$, etc., then $\rho: B_{2}(\ell) \rightarrow H_{2}(\pi(\ell))$.

We recall that a link $\ell$ is said to be splittable if we can find a decomposition $S^{n+2}=D_{1}^{n+2} \cup D_{2}^{n+2}$ such that some of the components $\left(\ell_{1}\right)$ of $\ell$ are in $D_{1}^{n+2}$ and the others $\left(\ell_{2}\right)$ are in $D_{2}^{n+2}$; we write $\ell=\ell_{1} \sqcup \ell_{2}$ for this spiitting.

If such decomposition is impossible the link is called unsplittable.

The sphere $S^{n+1}$ which is the common boundary of $D_{1}^{n+2}$ and $D_{2}^{n+2}$ will be called the splitting sphere.

PROPOSITION (3.7). If $\ell$ is a split link $\ell=\ell_{1} \sqcup \ell_{2}$ then

(a) $\pi(\ell)=\pi\left(\ell_{1}\right) * \pi\left(\ell_{2}\right)$,

(b) $B_{1}(\ell) \simeq B_{1}\left(\ell_{1}\right) \oplus \Lambda \oplus B_{1}\left(\ell_{2}\right)$,

(c) $B_{i}(\ell) \simeq B_{i}\left(\ell_{1}\right) \oplus B_{i}\left(\ell_{2}\right)$ for $2 \leq i \leq n$.

The statement on the fundamental group is a obvious application of van Kampen's theorem with the use of the splitting sphere.

For the homology, notice that $\hat{X}(\ell)$ can be obtained by a "equivariant connected sum" along the lifting of the separating sphere and so the statement follows from an application of the Mayer-Vietoris sequence.

Observe that the proposition guarantees that, from the point of view of the invariants we are studying, it is sufficient to analyze nonsplittable links.

For classical nonsplittable links $\ell, X(\ell)$ is a $K(\pi, 1)$ and $\hat{X}(\ell)$ is a $K(\hat{\pi}, 1)$; if its multiplicity is $m$, then $H_{2}(\pi(\ell)) \simeq Z^{m-1}$, which differs from the nonclassical case where $H_{2}(\pi(\ell))=0$.

COROLlaRY (3.8). If $\ell_{m}^{n}$ denotes the trivial link of dimension $n$ and multiplicity $m$, then

$$
B_{1}\left(\ell_{m}^{n}\right) \simeq \Lambda^{m-1} \simeq B_{n+1}\left(\ell_{m}^{n}\right) \quad \text { and } \quad B_{i}\left(\ell_{m}^{n}\right)=0 \quad \text { for } 2 \leq i \leq n .
$$

Given a split link $\ell=\ell_{1} \sqcup \ell_{2}$ and points $A$ and $B$ on component knots $k_{1}$ and $k_{2}$ of $\ell_{1}$ and $\ell_{2}$, respectively, we can choose a segment from $A$ to $B$ that crosses 
the splitting sphere only once (transversally) and intersects the link (transversally) only at its end points, namely, at $A$ and $B$.

Taking an $(n+1)$-dimensional tube along the segment, we obtain a connected sum $k=k_{1} \#_{c} k_{2}$ of the two knots $k_{1}$ and $k_{2}$ which can certainly be arranged so that the tube does not intersect the other components of the link.

Considering now $k$ and all the other embedded spheres of $\ell$ except $k_{1}$ and $k_{2}$, we get a link which we denote $\ell_{1} \#_{c} \ell_{2}$, which depends on $k_{1}, k_{2}$ and on the choice of the segment from $k_{1}$ to $k_{2}$.

Proposition (3.9). For a split link $\ell=\ell_{1} \cup \ell_{2}$ of dimension $n$, we have $B_{i}\left(\ell_{1} \#_{c} \ell_{2}\right) \simeq B_{i}\left(\ell_{1}\right) \oplus B_{i}\left(\ell_{2}\right)$ for $1 \leq i \leq n$.

The connected sum construction shows, as in the case of knots, that $X\left(\ell_{1} \#_{c} \ell_{2}\right)$ can be described, up to homotopy, as the union of $X\left(\ell_{1}\right)$ and $X\left(\ell_{2}\right)$, identifying a meridian of $k_{1}$ with a meridian of $k_{2}$ and preserving orientations. In $\hat{X}\left(\ell_{1}\right)$ and $\hat{X}\left(\ell_{2}\right)$, the meridians lift to copies of the real line and so $\hat{X}\left(\ell_{1} \#_{c} \ell_{2}\right)$ can be constructed from $\hat{X}\left(\ell_{1}\right)$ and $\hat{X}\left(\ell_{2}\right)$ identifying copies of the real line in each component. Now, the proposition follows by the Mayer-Vietoris sequence.

COROLlaRY (3.10). For a split link $\ell=\ell_{1} \cup \ell_{2}$ of dimension $n$, we have $B_{i}\left(\ell_{1} \#_{c_{1}} \ell_{2}\right) \simeq B_{i}\left(\ell_{1} \#_{c_{2}} \ell_{2}\right)$ for $1 \leq i \leq n$.

OBSERVATION. In reality, the spaces $X\left(\ell_{1} \#_{c_{1}} \ell_{2}\right)$ and $X\left(\ell_{1} \#_{c_{2}} \ell_{2}\right)$ are homotopy equivalent since both are homotopy equivalent to $X\left(\ell_{1}\right) \vee X\left(\ell_{2}\right)$, but in general the homotopy equivalence is not equivariant. Thus, the isomorphism obtained in the corollary in general is not included by a map.

From now on we use the notation $\ell_{1} \# \ell_{2}$ instead of $\ell_{1} \#_{c} \ell_{2}$; this is only consistent from the point of view of the modules $B_{i}$ 's.

PROPOSITION (3.11). If a group $G$ satisfies $H_{1}(G) \simeq Z^{m}$ and has a presentation of deficiency $m$, then $\mathrm{H}_{2}(G)=0$.

For the proof let $G=\left|g_{1}, g_{2}, \ldots, g_{m+r} ; S_{1}, S_{2}, \ldots, S_{r}\right|$ and $P_{1}=\bigvee_{i=1}^{m+r} S_{i}^{1}$.

The identification of $g_{i}$ with the element of $\pi_{1}\left(P_{1}\right)$ correspondent to the $i$ th circle allows the representation of each $S_{j}$ by a map $f_{j}: S^{1} \rightarrow P_{1}$.

Construct $P_{2}$ from $P_{1}$ by attaching $r$ 2-cells using the maps $\left\{f_{j}\right\}$.

Clearly $\pi_{1}\left(P_{2}\right)=G$.

A $K(G, 1)$ space can be obtained from $P_{2}$ by attaching cells of dimension 3 and higher and we have $H_{2}(G)=H_{2}(K(G, 1)) \simeq H_{2}\left(P_{2}\right)$.

Using now the homology sequence of the pair $\left(P_{2}, P_{1}\right)$ and the hypothesis one gets $H_{2}\left(P_{2}\right)=0$, proving the proposition.

PROPOSITION (3.12). If $(G, \alpha)$ is a strongly $m$-admissible pair, then $H_{2}(G)=$ $0=H_{2}(\hat{G}), H_{1}(\hat{G}) \otimes_{\Lambda} Z \simeq Z^{m-1}$ and $H_{1}(\hat{G})$ is pseudo null free.

By the last proposition we already have $H_{2}(G)=0$.

Constructing $P_{2}$ as before we have a surjection $\alpha: \pi_{1}\left(P_{2}\right) \rightarrow Z$ and a corresponding covering $\hat{P}_{2} \rightarrow P_{2}$.

$P_{2}$ has a $\Lambda$-free chain complex $C_{2}\left(\hat{P}_{2}\right) \stackrel{d_{2}}{\rightarrow} C_{1}\left(\hat{P}_{2}\right) \rightarrow C_{0}\left(\hat{P}_{2}\right)$ and $H_{2}\left(\hat{P}_{2}\right)$ is the kernel of $d_{2}$ and so is a free $\Lambda$-module. 
Using now the Cartan-Leray spectral sequence of $\hat{P}_{2} \rightarrow P_{2}$ and the fact that $H_{2}\left(P_{2}\right)=0$, we obtain that $H_{1}\left(\hat{P}_{2}\right) \otimes_{\Lambda} Z \simeq Z^{m-1}$ and that $H_{2}\left(\hat{P}_{2}\right)$ is of type $K$. Since $H_{1}(\hat{G}) \simeq H_{1}\left(\hat{P}_{2}\right), H_{1}(\hat{G}) \otimes_{\Lambda} Z \simeq Z^{m-1}$. Since $H_{2}\left(\hat{P}_{2}\right)$ is of type $K$, it is $\Lambda$-torsion (Corollary 1.3 of $[\mathbf{L . 1}]$ ) and so it must be zero since we have seen above that it is a free $\Lambda$-module. The Hopf surjection $H_{2}\left(\hat{P}_{2}\right) \rightarrow H_{2}(\hat{G})$ implies that $H_{2}(\hat{G})=0$.

From the chain complex above, we get a free resolution $0 \rightarrow C_{2}\left(\hat{P}_{2}\right) \rightarrow Z_{1}\left(\hat{P}_{2}\right) \rightarrow$ $H_{1}\left(\hat{P}_{2}\right) \rightarrow 0$, so $H_{1}\left(\hat{P}_{2}\right)$ has homological dimension $\leq 1$. As a result it is pseudo null free by Proposition 1.18 .

PROPOSITION (3.13). Let $B$ be a pseudo null free $\Lambda$-module such that $T(B)$ is of type $K$ and $B \otimes_{\Lambda} Z \simeq Z^{m-1}$. Then there is a strongly $m$-admissible pair $(G, \alpha)$ with $H_{1}(\hat{G})=B$.

We recall that $\varepsilon: \Lambda \rightarrow Z$ denotes the canonical augmentation $\varepsilon(t)=1$.

Since $B$ is pseudo null free and $B \otimes_{\Lambda} Z \simeq Z^{m-1}$, after row and column operations in a presentation of $B$, we can get a presentation of the form $0 \rightarrow \Lambda^{r} \stackrel{\lambda}{\rightarrow}$ $\Lambda^{r+m-1} \rightarrow B \rightarrow 0$. Here, $\lambda=\left(\lambda_{i j}\right)$ is a $(r+m-1) \times r$ matrix over $\Lambda$ such that $\varepsilon(\lambda)=\left[I_{r \times r}, 0_{r \times(m-1)}\right]^{\prime}$, where $I_{r \times r}$ is the identity matrix and $0_{r \times(m-1)}$ is the zero matrix. We write $\lambda_{i j}(t)=\sum_{k} a_{i j k} t^{k}$ where $i=1,2, \ldots, r$ and $j=1,2, \ldots, r+m-1$.

Define the group $G$ by the presentation

$$
G=\left|x_{1}, x_{2}, \ldots, x_{n}, x_{n+1}, x_{n+2}, \ldots, x_{n+m-1}, \tau: R_{1}, R_{2}, \ldots, R_{r}\right|
$$

where

$$
R_{i}=\left[\prod_{(1)}\left(\tau^{k} x_{j} \tau^{-k}\right)^{a_{i j k}}\right] \cdot\left[\prod_{(2)}\left(\tau^{k-1} x_{j} \tau^{-k}\right)^{a_{i j k}}\right] \quad \text { for } i=1,2, \ldots, r
$$

and the indices (1) are $k=-\infty, \infty$ and $j=1,2, \ldots, r$ and the indices (2) are $k=-\infty, \infty$ and $j=r+1, r+2, \ldots, r+m-1$.

The products should be carried out in a lexicographic ordering of $(j, k)$.

Notice that $a_{i j k}=0$ for $|k|>k_{0}$, so the presentation is finite.

A presentation for $H_{1}(G)$ is then in additive notation

$$
\begin{aligned}
\mid X_{1}, X_{2}, \ldots, X_{r}, X_{r+1}, T:\left\{\sum_{j=1}^{r}\left(\sum_{k} a_{i j k}\right) X_{j}\right\} \\
+\left\{\sum_{j=r+1}^{r+m-1}\left[\sum_{k} a_{i j k}\left(X_{j}-T\right)\right]\right\}, i=1,2, \ldots, r \mid
\end{aligned}
$$

and since $\sum_{k} a_{i j k}=\varepsilon\left(\lambda_{i j}\right)=0$ or 1 conveniently we get $X_{j}=0$ for $j=1,2, \ldots, r$ and so $H_{1}(G)=\left|X_{r+1}, X_{r+2}, \ldots, X_{r+m-1}, T:\right| \simeq Z^{m}$.

From this we get weight $(G) \geq m$.

Observe now that the quotient of $G$ by $\langle\tau\rangle$ is a free group on $x_{r+1}, x_{r+2}, \ldots$, $x_{r+m-1}$, that is, we have a exact sequence $0 \rightarrow\langle\tau\rangle \rightarrow G \rightarrow F(m-1) \rightarrow 0$, so weight $(G)$ must be $m$.

Consider now the isomorphism $\Omega: H_{1}(G) \rightarrow Z^{m}$ that sends $\left\{x_{1}, x_{2}, \ldots, x_{m-1}\right.$, $T\}$ to the canonical generators of $Z^{m}$ and define $\alpha: G \rightarrow Z$ to be the composition 
$\Sigma \cdot \Omega \cdot A \cdot$ where $A$ abelianization and $\Sigma$ is sum of coordinates. Let $\hat{G}$ be the kernel of $\alpha$ and notice that for $i=r+1, \ldots, r+m-1, \alpha\left(x_{i}\right)=\alpha(\tau)$ is a generator of $Z$, and so we can use them to define a $\Lambda$ structure on $H_{1}(\hat{G})$.

We have that $(G, \alpha)$ is a strongly $m$-admissible pair and we need to prove that $H_{1}(\hat{G}) \simeq B$.

For this, observe that the collection $\left\{\tau^{k} x_{j} \tau^{-k}\right.$ and $\left.\tau^{k} x_{j}^{-k}\right\}$ form a set of generators for $\hat{G}$. It follows that a presentation for $\hat{G}$ is

$$
\left|\left\{x_{j k}\right\}_{k=-\infty, \infty}^{j=1, r+m-1}: R(i, \ell), i=1,2, \ldots, r, \ell \in Z\right|
$$

where

$$
R(i, \ell)=\left[\prod_{(1)} x_{j, k+\ell}^{a_{i j k}}\right] \cdot\left[\prod_{(2)} x_{j, k+\ell}^{a_{i j k}}\right]
$$

and we have $x_{j k}=\tau^{k} x_{j} \tau^{-k}$ for $j=1,2, \ldots, r$ and $x_{j k}=\tau^{k} x_{j}^{-k}$ for $j=r+1, \ldots$, $r+m-1$. A presentation of $H_{1}(\hat{G})$ is then

$$
\left|\left\{X_{j k}\right\}: \sum_{j=1}^{r} a_{i j k} X_{j, k+\ell}+\sum_{j=r+1}^{r+m-1} a_{i j k} X_{j, k+\ell}=0, i=1,2, \ldots, r, \ell \in Z\right| .
$$

If we write $X_{j k}=t^{k} X_{j}$ using the $\Lambda$-module structure of $H_{1}(\hat{G})$ we get a $\Lambda$-module presentation.

$$
\left|X_{j}: t^{\prime}\left(\sum_{j} \lambda_{i j} X_{j}\right), i=1,2, \ldots, r, \ell \in Z\right|
$$

which is a presentation of $B$.

PROPOSITION (3.14). Let $(G, \alpha)$ be an $m$-admissible pair with $H_{2}(G)=0$ and $F$ a pseudo null free $\Lambda$-module with a epimorphism $\rho: F \rightarrow H_{2}(\hat{G})$. Then there is a connected 3-dimensional complex $P$ with $\pi_{1}(P) \simeq G$ such that, for the infinite cyclic covering $\hat{P}$ corresponding to $\hat{G}, H_{3}(\hat{P})=0, H_{2}(\hat{P}) \simeq F$ and $\rho$ corresponds to the Hopf homomorphism.

If $(G, \alpha)$ is strongly $m$-admissible (in this case $F$ is necessarily zero), $P$ may be actually 2 -dimensional.

The proof follows the steps of Lemma 9.3 of $[\mathbf{L . 1}]$ with the necessary adjustments.

Choose a presentation of $G=\left|x_{1}, x_{2}, \ldots, x_{n}: r_{1}, r_{2}, \ldots, r_{s}\right|$ and take a wedge of $n$ circles, adjoining 2-cells using the relations $r_{j}$. Let $P_{2}$ be this space and call $\hat{P}_{2}$ the cyclic covering associated with $\hat{G}=$ kernel of $\alpha$.

$H_{2}\left(\hat{P}_{2}\right)$ is a free $\Lambda$-module because it is the kernel of $\partial: C_{2}\left(\hat{P}_{2}\right) \rightarrow C_{1}\left(\hat{P}_{2}\right)$ and $C_{2}\left(\hat{P}_{2}\right)$ is free. From Hopf's theorem, we have the exact sequence $\pi_{2}\left(\hat{P}_{2}\right) \stackrel{a}{\rightarrow}$ $H_{2}\left(\hat{P}_{2}\right) \stackrel{\rho}{\rightarrow} H_{2}(\hat{G}) \rightarrow 0$. Since $H_{2}\left(\hat{P}_{2}\right)$ is free and $\rho: F \rightarrow H_{2}(\hat{G})$ is onto, we may add some 2 -spheres to $P_{2}$ if necessary to get a surjective homomorphism $\varphi$ giving 
a commutative diagram

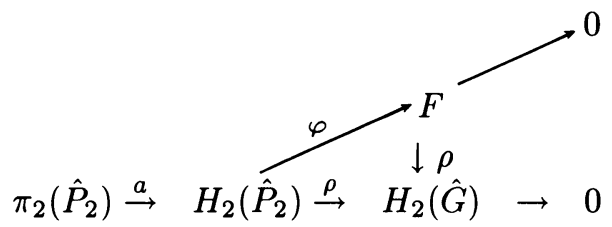

Since $F$ is pseudo null free, it has homological dimension one by Proposition (1.18); therefore, $\operatorname{ker} \varphi$ is a free $\Lambda$-module inside $a\left(\pi_{2}\left(\hat{P}_{2}\right)\right)$.

We choose a basis for ker $\varphi$ and represent its elements by maps $S^{2} \rightarrow P_{2}$. Construct $P$ from $P_{2}$ by attaching 3-cells using these maps. It follows that $H_{2}(\hat{P}) \simeq$ $F$ because $\varphi$ was surjective and its kernel is killed by the attaching of the 3-cells. $H_{3}(P)=0$ since the boundary operator $C_{3}(\hat{P}) \rightarrow C_{2}(\hat{P})=C_{2}\left(\hat{P}_{2}\right)$ is injective.

PROPOSITION (3.15). Let $P$ be a d-dimensional complex with $H_{2}\left(\pi_{1}(P)\right)=0$ and $\left(\pi_{1}(P), \alpha\right)$ an $m$-admissible pair.

Let $\hat{P}$ the covering of $P$ corresponding to ker $\alpha$ and suppose that $H_{i}(\hat{P})$ is of type $K$ for $i \geq 2$.

If $d=2$, assume that $H_{2}(P)=0$.

Then $P$ embeds in $R^{n+3}$ where $n \geq \sup \{2,2 d-3\}$ and if we call $Y$ the boundary of a regular neighborhood of $P$ in $R^{n+3}$ then

(1) $\pi_{1}(Y) \simeq \pi_{1}(P)$.

(2) $H_{0}(Y) \simeq Z \simeq H_{n+2}(Y) ; H_{1}(Y) \simeq Z^{m} \simeq H_{n+1}(Y)$ and $H_{i}(Y)=0$ for $i \neq 0,1, n+1, n+2$.

(3) If $\hat{Y}$ is the covering of $Y$ corresponding to ker $\alpha$, then $H_{i}(\hat{Y}) \simeq H_{i}(\hat{P})$ for $i \leq n+1-d$.

(4) If $n=2 d-3$ and $H_{d-1}(\hat{P})$ is pseudo null, then $H_{d-1}(\hat{Y})$ is pseudo null.

If $d \geq 3$, consider the exact sequence derived from the Cartan-Leray spectral sequence of $\hat{X} \rightarrow X$ :

$$
H_{2}(\hat{P}) \stackrel{(t-1)}{\rightarrow} H_{2}(\hat{P}) \rightarrow H_{2}\left(P_{2}\right) \rightarrow H_{1}(\hat{P}) \rightarrow H_{1}(\hat{P}) \rightarrow H_{1}(P) \rightarrow \cdot
$$

Since $H_{i}(\hat{P})$ are of type $K$ for $i \geq 2$, we get $H_{i}(P)=0$, and the imbeddability follows from a theorem of Van Kampen. [V.2].

If $N$ is a regular neighborhood of $P$ in $R^{n+3}$, then $Y$ is a deformation retract of $N-P$, and therefore $\pi_{i}(N, Y)=\pi_{i}(N, N-P)=0$ for $i<n+3-d$ by general position. Since $n+3-d \geq 3$, we have $\pi_{1}(Y) \simeq \pi_{1}(N)$ which implies (1) because $N$ is homotopy equivalent to $P$.

The inclusion $i: Y \rightarrow N$ induces an isomorphism in $\pi_{1}$ so we get $\hat{Y} \stackrel{\hat{i}}{\rightarrow} \hat{N}$. For $i \leq n+3-d$, we have $\pi_{i}(\hat{N}, \hat{Y})=0$, that is, $(\hat{N}, \hat{Y})$ is $(n+2-d)$-connected. By the Hurewicz theorem, $H_{i}(\hat{Y}) \simeq H_{i}(\hat{N})$ for $i \leq n+1-d$, and so $H_{i}(\hat{Y}) \simeq H_{i}(\hat{P})$ for $i \leq n+1-d$, which is $(3)$.

Since $n \geq 2 d-3$, we get $n+2-d \geq(n+1) / 2$. Hence, $H_{i}(Y)=0$ for $2 \leq i \leq$ $(n+2) / 2$ because $H_{i}(Y) \simeq H_{i}(P)$ for $i \leq(n+2) / 2$ and $H_{i}(P)=0$ for $i \geq 2$. We have $H_{0}(Y) \simeq Z$ and $H_{2}(Y) \simeq H_{1}(P) \simeq Z^{m}$, so by Poincaré duality we obtain (2).

To prove (4), consider the homology sequence of $(\hat{N}, \hat{Y}) \cdots H_{d}(\hat{N}, \hat{Y}) \rightarrow H_{d-1}(\hat{Y})$ $\rightarrow H_{d-1}(\hat{N}) \rightarrow \cdots$. Since $H_{d-1}(\hat{N})$ is finite it is sufficient to prove that $H_{d}(\hat{N}, \hat{Y})$ is finite. 
Since $n=2 d-3,(d-1) \geq 2$ and $H_{i}(\hat{P})$ is of type $K$ for $i \geq 2$, the universal coefficient spectral sequence gives a short sequence $0 \rightarrow e^{2}\left(H_{d-2}(\hat{P})\right) \rightarrow H_{e}^{d}(P) \rightarrow$ $e^{1}\left(H_{d-1}(\hat{P})\right) \rightarrow 0$. We have $H_{d-1}(\hat{P})$ finite (or pseudo null), so $e^{1}\left(H_{d-1}(\hat{P})\right)=0$. This implies $H_{e}^{d}(\hat{P}) \simeq e^{2}\left(H_{d-1}(\hat{P})\right)$, which is pseudo null by Proposition (1.6). Since $H_{d}(\hat{N}, \hat{Y}) \simeq H_{e}^{d}(\hat{N}) \simeq H_{e}^{d}(\hat{P})$, we have that $H_{d}(\hat{N}, \hat{Y})$ is finite, and so the proposition follows.

Consider the manifold $Y$ constructed above. Let $\alpha_{1}, \alpha_{2}, \ldots, \alpha_{m}$ in $\pi_{1}(Y)$ with $\left\langle\alpha_{1}, \ldots, \alpha_{m}\right\rangle=\pi_{1}(Y)$ and $\bar{\alpha}_{1}, \ldots, \bar{\alpha}_{n}$ a base for $H_{1}(Y)$. Represent $\alpha_{1}, \ldots, \alpha_{m}$ by disjoint embedded circles $S_{1}, S_{2}, \ldots, S_{m}$. They have trivial normal bundles since $Y$ is orientable and so there are tubular neighborhoods $T_{1}, T_{2}, \ldots, T_{m}$ diffeomorphic to $S^{1} \times D^{n+1}$.

Define $X=Y-\bigcup_{i=1}^{m} T_{i}$. Then by general position, $\pi_{i}(Y, X)=0$ for $i \leq n$, and, in case $n \geq 2, \pi_{1}(X) \simeq \pi_{1}(Y), H_{i}(X) \simeq H_{i}(Y)$ and $H_{i}(\hat{X}) \simeq H_{i}(\hat{Y})$ for $i<n$.

The boundary of $X$ is diffeomorphic to $\bigcup_{j=1}^{m}\left(S^{1} \times S^{n}\right)_{j}$ and we attach to $X \cdot m$ copies of $D^{2} \times S^{n}$ via diffeomorphisms on the boundaries. Let $\Sigma$ be the resulting manifold.

It follows from van Kampen's theorem (and the fact that $(G, \alpha)$ is $m$-admissible) that $\Sigma$ is simply-connected. Also,

$$
H_{i}(\Sigma, X) \simeq H_{i}\left(\bigcup_{j=1}^{m}\left(\left(D^{2}, S^{1}\right) \times S^{n}\right)_{j}\right)
$$

so

$$
H_{2}(\Sigma, H) \simeq H_{n+1}(\Sigma, X) \simeq H_{n+2}(\Sigma, X) \simeq Z^{m}
$$

and $H_{i}(\Sigma, X)=0$ for $i \neq 2, n+1$ and $n+2$. By the sequence of the pair $(\Sigma, X)$ and Whitehead's theorem, $\Sigma$ is a homology $(n+2)$-sphere. If $n \geq 3$ we can change $\Sigma$ to $S^{n+2}$ without changing $X$.

Considering now $K_{j}^{n}=0 \times S_{j}^{n} \subset D^{2} \times S_{j}^{n} \subset S^{n+2}$ we have a link $\ell$ in $S^{n+2}$ whose complement is $X$, meridional curves determined by $\alpha_{1}, \alpha_{2}, \ldots, \alpha_{m}, B_{i}(\ell) \simeq H_{i}(\hat{P})$ for $i \leq n+1-d,(X(\ell)) \simeq \pi_{1}(P)$ and $\hat{\pi}(X(\ell)) \simeq \pi_{1}(\hat{P})$.

The above construction in connection with the last two propositions gives us certain links which are basic in the realization work. We collect the results in the following propositions.

Proposition (3.16). Let $(G, \alpha)$ be a strongly $m$-admissible pair. Then there is a link $\ell: \bigcup_{i=1}^{m} S_{i}^{2} \rightarrow K^{4}\left(K^{4}\right.$ is a homotopy 4-sphere) with $\pi_{1}(\ell)=G$ such that a choice of orientation and meridians reproduces the surjection $\alpha: G \rightarrow Z$. Moreover, $B_{2}(\ell)=0$.

Proposition (3.17). Let $(G, \alpha)$ be an m-admissible pair with $\mathrm{H}_{2}(G)=0=$ $H_{2}(\hat{G})$. Then there is a link $\ell: \bigcup_{i=1}^{m} S_{i}^{3} \rightarrow S^{5}$ such that $\pi(\ell)=G$ such that a choice of orientations and meridians reproduces the surjection $\alpha: G \rightarrow Z$. Moreover, $B_{2}(\ell)$ is pseudo null.

PROPOSITION (3.18). Let $(G, \alpha)$ be an m-admissible pair with $\mathrm{H}_{2}(G)=0$ and $\rho: F \rightarrow H_{2}(\hat{G})$ a surjection where $F$ is pseudo null free of type $K$. Then there is a link $\ell: \bigcup_{i=1}^{m} S_{i}^{n} \rightarrow S^{n+2}(n \geq 4)$ with $\pi(\ell)=G$ such that a choice 
of orientations and meridians reproduces the surjection $\alpha: G \rightarrow Z$. Moreover, $B_{2}(\ell) \simeq F, B_{i}(\ell)=0$ for $3 \leq i \leq n-2$ and $\rho$ is the Hopf homomorphism.

PROPOSITION (3.19). Let $(G, \alpha)$ be a strongly $m$-admissible pair such that $G$ has a presentation

$$
G=\left|x_{1}, \ldots, x_{t+m}: r_{1}, \ldots, r_{t}\right|
$$

with

$$
\left\langle x_{t+1}, x_{t+2}, \ldots, x_{t+n}\right\rangle=G \quad \text { and } \quad r_{j}\left(x_{1}, \ldots, x_{t}, 1,1, \ldots, 1\right)=x_{j},
$$

$j=1,2, \ldots, t$.

Then there is a link $\ell: \bigcup_{i=1}^{m} S_{i}^{2} \rightarrow S^{4}$ with $\pi(\ell)=G$ such that a choice of orientations and meridians reproduces the surjection $\alpha: G \rightarrow Z$. Moreover, $B_{2}(\ell)=0$.

Let $V_{0}=\#_{j=1}^{t+m}\left(S^{1} \times S^{3}\right)_{j} . \pi_{1}\left(V_{0}\right)$ is free on $(t+m)$ generators $x_{1}, \ldots, x_{t+m}$ which are represented by loops in $\left(S^{1} \times S^{3}\right)_{j}$. If we construct $V$ from $V_{0}$ by doing surgery on embeddings $f_{k}: S^{1} \times D^{3} \rightarrow V_{0}$ representing $r_{k}, k=1,2, \ldots, t$, we obtain $\pi_{1}(V) \simeq G$.

Now, $\left\langle x_{t+1}, \ldots, x_{t+m}\right\rangle=G$ so, by doing surgery on $V$ using maps $g_{i}: S^{1} \times D^{3} \rightarrow$ $V$ representing $x_{t+1}, i=1,2, \ldots, m$, we produce a homotopy 4 -sphere $\Sigma$ with a link $\ell$ given by $g_{i}\left(\{*\} \times S^{2}\right)$ whose complement is $V$. We would be done, except that $\Sigma$ may not be $S^{4}$.

If we do surgery on $V_{0}$ first using $\left\{g_{i}\right\}, i=1,2, \ldots, m$, we obtain

$$
V_{1}=\left(\underset{j=1}{\#}\left(S^{1} \times S^{3}\right)_{j} \#\left(\underset{j=t+1}{\#} S_{i}^{t+m}\right)=\underset{j=1}{\#}\left(S^{1} \times S^{3}\right)_{j}\right.
$$

The relations $r_{j}$ become $r_{j}\left(x_{1}, \ldots, x_{t}, 1,1, \ldots, 1\right)=x_{j}$, and the embeddings $\bar{f}_{k}$ : $S^{1} \times D^{3} \rightarrow V_{1}$ induced by $f_{k}$ are homotopic to the embeddings of $\left(S^{1} \times D^{3}\right)_{k} \subset V_{1}$.

In fact, by general position, each $\bar{f}_{k}$ is isotopic to the embeddings of $\left(S^{1} \times D^{3}\right)_{k}$ or some twisting of it. In the latter case, we can adjust the embedding $f_{k}$ by untwisting its normal frame so that $\bar{f}_{k}$ becomes isotopic to the embeddings $\left(S^{1} \times D^{3}\right)_{k}$.

It now follows that surgery on $\bar{f}_{k}$ essentially changes the $\left(S^{1} \times S^{3}\right)_{k}$ to $S_{k}^{3}$, so the resulting manifold after these surgeries is $S^{4}$ and the proposition follows.

Notice that the group constructed in Proposition (3.13) satisfies the conditions of Proposition (3.19). This gives the next corollary.

COROLlaRY (3.20). Let $B$ be a pseudo null free $\Lambda$-module such that $T(B)$ is of type $K$ and $B \otimes_{\Lambda} Z \simeq Z^{m-1}$. Then there is a link $\ell: \bigcup_{i=1}^{m} S_{i}^{2} \rightarrow S^{4}$ with $\pi(\ell)=G$ such that a choice of orientations and meridians give us $B_{1}(\ell) \simeq B$ and $B_{2}(\ell)=0$.

THEOREM (3.21). Let $n \geq 2,(G, \alpha)$ an $m$-admissible pair and $\left(B_{2}, \ldots, B_{n}\right)$ a sequence of $\Lambda$-modules of type $K$; denote by $\hat{G}$ the kernel of $\alpha$ and $B_{1}=H_{1}(\hat{G})$.

Let $\rho: B_{2} \rightarrow H_{2}(\hat{G})$ be a surjection and suppose that collection $\left(B_{1}, B_{2}, \ldots, B_{n}\right)$ satisfies the necessary conditions $(\mathrm{c}),(\mathrm{d}),(\mathrm{e}),(\mathrm{f}),(\mathrm{g}),(\mathrm{h}),(\mathrm{i})$ and $(\mathrm{j})$ of Theorem 2.20 .

Assume further the additional conditions:

(1) $B_{2}$ has a pseudo null free summand $F_{2}$ such that $\rho\left(F_{2}\right)=H_{2}(\hat{G})$. 
(2) If $n=2, G$ has a presentation of the form $\left|x_{1}, x_{2}, \ldots, x_{t+m}: r_{1}, \ldots, r_{t}\right|$ such that $\left\langle x_{t+1}, \ldots, x_{t+m}\right\rangle=G$ and the relations $r_{j}, j=1,2, \ldots, k$ satisfy the conditions $r_{j}\left(x_{1}, \ldots, x_{k}, 1,1, \ldots, 1\right)=x_{j}$.

Then there is a link $\ell: \bigcup_{i=1}^{m} S_{i}^{n} \rightarrow S^{n+2}$ with $\pi_{1}(X(\ell))=G$ and if we denote the covering associated to the kernel of $\alpha$ by $\hat{X}(\ell)$, we have

$$
\begin{aligned}
& B_{j} \simeq H_{j}(\hat{X}(\ell)) \text { for } 1 \leq i \leq[n / 2] \text { or } i=n . \\
& N\left(B_{j}\right) \simeq N\left(H_{j}(\hat{X}(\ell))\right) \text { and } F N\left(B_{j}\right)=F N\left(H_{j}(\hat{X}(\ell))\right) \text { for } j=1,2, \ldots, n .
\end{aligned}
$$

REMARK. If we allow links in homotopy spheres, condition (2) can be eliminated.

Observe that the conditions on $G$ imply that $H_{1}(\hat{G}) \otimes_{\Lambda} Z \simeq Z^{m-1}$ and rank $H_{1}(\hat{G})=m-1$.

For $n=2$, this is exactly Proposition 3.19.

For $n=2 q \geq 4$, consider the knot $k_{q}$ given by Proposition (3.5) where $A$ is taken to be $N\left(B_{q}\right)$. Then, for $i=2,3, \ldots,(q-1)$, consider the knots $k_{i}$ given by Proposition (3.3) where $A$ is taken to be $B_{2} / F_{2}, B_{3}, \ldots, B_{q-1}$ respectively.

Let $k$ be the connected sum of $k_{2}, k_{3}, \ldots, k_{q}$ so $\pi_{1}(X(k)) \simeq Z, B_{1}(k)=0$, $B_{2}(k) \simeq B_{2} / F_{2}, B_{i}(k) \simeq B_{i}$ for $3 \leq i \leq(q-1)$ and $B_{q}(k) \simeq N\left(B_{q}\right)$.

Now, by using Proposition (3.18) with $F=F_{2}$, we obtain a link $\ell_{1}$ with $B_{1}\left(\ell_{1}\right)=$ $B_{1}, B_{2}\left(\ell_{1}\right)=F_{2}$ and $\pi\left(\ell_{1}\right)=G$.

It is easy to check that any link of the form $\ell=\ell_{1} \# k$ satisfies the conclusion of the theorem for the even case.

If $n=2 q-1 \geq 3$, consider the knot $h_{q}$ given by Proposition (3.4) where $F$ is taken to be $F N\left(B_{q}\right)$. For $i=2,3, \ldots,(q-1)$ consider the knots $h_{i}$ given by Proposition (3.3) where, as before, $A$ is taken to be $B_{2} / F_{2}, B_{3}, \ldots, B_{q-1}$, respectively.

Let $h$ be the connected sum of $h_{2}, h_{3}, \ldots, h_{q}$ so that $\pi_{1}(X(h)) \simeq Z, B_{1}(h)=0$, $B_{2}(h) \simeq B_{2} / F_{2}, B_{i}(h) \simeq B_{i}$ for $3 \leq i \leq q-1$, and $B_{q}(h) \simeq F N\left(B_{q}\right)$.

Again, it is easy to check that any link $m=\ell_{1} \# h$ satisfies the conclusions of the theorem in this last case.

The next result is a realization theorem concerning only the homology.

THEOREM (3.22). Let $n \geq 2$ and $\left(B_{1}, B_{2}, \ldots, B_{n}\right)$ a sequence of $\Lambda$-modules satisfying the necessary conditions $(\mathrm{a})$ to $(\mathrm{j})$ of Theorem (2.20). In addition, assume that $N\left(B_{1}\right)=0$.

Then there is a link $\ell: \bigcup_{i=1}^{m} S_{i}^{n} \rightarrow S^{n+2}$ such that

$B_{i}(\ell) \simeq B_{i}$ for $1 \leq i<[n / 2]$ and $i=n$.

$N\left(B_{i}(\ell)\right) \simeq N\left(B_{i}\right)$ and $F N\left(B_{i}(\ell)\right) \simeq F N\left(B_{i}\right)$ for $[n / 2] \leq j \leq(n-1)$. $B_{1}$.

By Proposition (3.13), there is a strongly $m$-admissible pair $(G, \alpha)$ with $H_{1}(\hat{G}) \simeq$

Proceeding as in Theorem (3.21) (and taking $F=0$ in Proposition (3.18), the result follows.

4. Applications. This section contains some results about the modules $B_{i}(\ell)$ for links of particular type.

(4.1) Fibered links.

DEFinition. A link $\ell: \bigcup_{i=1}^{m} K_{i}^{n} \rightarrow S^{n+2}$ is fibered if there is a smooth map $\phi: S^{n+2} \rightarrow R^{2}$ satisfying:

(i) 0 is a regular value and $L=\operatorname{im} \ell=\phi^{-1}(0)$,

(ii) $\phi /|\phi|: S^{n+2}-L \rightarrow S^{1}$ is a smooth fibration. 
If $X$ is a complement of a suitable tubular neighborhood of $L$, then $X$ fibers over $S^{1}$. A fixed fiber $F$ of this fibration can be used as a Seifert manifold for the link in the construction of $\hat{X}$ so that $\hat{X}$ is homeomorphic to $F \times R$.

We conclude that $H_{*}(\hat{X}(\ell)) \simeq H_{*}(F)$ which is finitely generated over $Z$, and hence

PROPOSITION (4.1.1). If $\ell$ is a fibered link, $\operatorname{rank}_{\Lambda} B_{i}(\ell)=0$.

COROLLARY (4.1.2). If $\ell$ is a link of dimension $n \geq 2$ and multiplicity $m \geq 2$, $\ell$ is not fibered.

For the proof, just remember that in this situation $\operatorname{rank}_{\Lambda} B_{1}(\ell)=(m-1)$.

(4.2) Boundary links.

DEFinition. A link $\ell: \bigcup_{j=1}^{m} K_{j}^{n} \rightarrow S^{n+2}$ is called a boundary link if there are $m$ disjoint orientable manifolds $M_{1}, \ldots, M_{m}$ in $S^{n+2}$ with $\partial M_{j}=\ell\left(K_{j}^{n}\right)$.

Proposition (4.2.1). If $\ell: \bigcup_{i=1}^{m} K_{i}^{n} \rightarrow S^{n+2}$ is a boundary link, then $B_{1}(\ell) \simeq \Lambda^{m-1} \oplus T\left(B_{1}(\ell)\right)$ and $B_{n}(\ell)$ is pseudo null free.

We have disjoint manifolds $M_{i} \subset S^{n+2}, i=1,2, \ldots, m$, with $\partial M_{i}=L_{i}$ (= $\left.\ell\left(K_{i}^{n}\right)\right)$. Adjust a tubular neighborhood $V_{i}$ of each $L_{i}$ and a tubular neighborhood of $M_{i}$ to get a $(n+2)$-dimension manifold $R_{i} \subset S^{n+2}$ such that $R_{i}$ is homeomorphic to $M_{i} \times[-1,1]$ with $M_{i}^{n+1}$ in its interior.

Consider $X=X(\ell)$ obtained by removing smaller tubular neighborhood $W i \subset$ $V_{i}$ of each $L_{i}$. Let $T_{i}=X(\ell) \cap R_{i}$ and $Q=X-\left(\bigcup_{i=2}^{m} T_{i}\right)$ (notice that $\left.T_{1} \subset Q\right)$.

$\hat{X}$ can be constructed by cutting $X$ along $M_{i}$ in the same fashion as one does to construct the universal abelian covering for knots (in fact, the cutting is in the part of $M_{i}$ inside $X$ ).

We observe that the spaces $Q, T_{2}, \ldots, T_{m}$ lift to connected spaces $\hat{Q}, \hat{T}_{2}, \ldots, \hat{T}_{m}$ inside $\hat{X}$ because each one of them has a "gluing cut" in its interior, or, alternatively because they contain a meridian which goes to a generator of $Z$ by the map $\alpha: \pi_{1}(X) \rightarrow Z$.

Notice that the boundary of $T_{i}$ has two connected components, one homeomorphic to $S^{n} \times S^{1}$ and the other to the double of $M_{i}$. The first component lifts to $S^{n} \times R$, while the second lifts to a disjoint union of copies of itself which we denote by $\hat{N}_{i}$ (notice that $\hat{N}_{i}$ is a manifold with infinitely many connected components).

Consider now the sequence of the pairs $(\hat{X}, \hat{Q})$ and $(\hat{T}, \hat{N})=\left(\bigcup_{i=2}^{m} \hat{T}_{i}, \bigcup_{i=2}^{m} \hat{N}_{i}\right)$, which are related by the following commutative diagram:

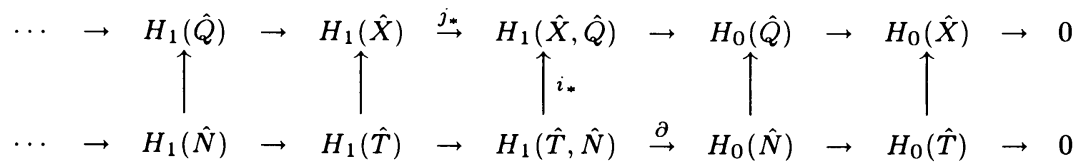

We have

$$
B_{1}(\ell)=H_{1}(\hat{X}) \stackrel{j_{*}}{\rightarrow} H_{1}(\hat{X}, \hat{Q}) \simeq H_{1}(\hat{T}, \hat{N})
$$

with $j_{*}$ surjective. Also, $\partial: H_{1}(\hat{T}, \hat{N}) \rightarrow H_{0}(\hat{N}) \simeq \Lambda^{m-1}$ satisfies image $\partial=$ $(t-1) \cdot H_{0}(\hat{N}) \simeq \Lambda^{m-1}$. Therefore $j_{*} i_{*}^{-1} \partial: B_{1}(\ell) \rightarrow \Lambda^{m-1}$ is a surjection, and $B_{1}(\ell) \simeq \Lambda^{m-1} \oplus T\left(B_{1}(\ell)\right)$. 
Now we recall that $B_{1}(\ell) \simeq(t-1) C_{1}(\ell) \simeq C_{1}(\ell)$ and so $C_{1}(\ell) \simeq \Lambda^{m-1} \oplus$ $T\left(C_{1}(\ell)\right)$. Finally $B_{n} \simeq e^{1}\left(\bar{C}_{1}\right) \simeq e_{1}\left(T\left(\bar{C}_{1}\right)\right)$ which is pseudo null free.

We propose next a generalization of the concept of boundary links.

DEFINITION. Let $i$ be a surjective function from $\{1,2, \ldots, m\}$ to $\{1,2, \ldots, r\}$. A link $\ell: \bigcup_{i=1}^{m} K_{i}^{n} \rightarrow S^{n+2}$ is said of type $i$ if there are disjoint oriented manifolds $W_{1}, W_{2}, \ldots, W_{r}$ such that $\partial W_{j}=\bigcup_{i(s)=j} \ell\left(K_{s}^{n}\right)$.

Clearly every link is of type $\{1,2, \ldots, m\} \rightarrow\{1\}$ and boundary links are those which are of type $\{1,2, \ldots, m\} \rightarrow\{1,2, \ldots, m\}$.

Using a similar proof as in the last proposition, we have

PROPOSITION (4.2.2). If $\ell: \bigcup_{i=1}^{m} K_{i}^{n} \rightarrow S^{n+2}$ is a link of type $i:\{1, \ldots, m\}$ $\rightarrow\{1,2, \ldots, r\}$, then $B_{1}(\ell)$ has a free summand of $\operatorname{rank}(r-1)$.

PROPOSITION (4.2.3). Given a collection of $\Lambda$-modules satisfying the conditions of Theorem (3.22) and the extra conditions $B_{1} \simeq \Lambda^{m-1} \oplus$ Torsion and $B_{n}$ pseudo null free.

Then there is a boundary link (in fact a totally splittable link) $\ell$ with

(i) $B_{i}(\ell) \simeq B_{i}$ for $1 \leq i \leq[n / 2]$ and $i=n$.

(ii) $N\left(B_{i}(\ell)\right) \simeq N\left(B_{i}\right)$ and $F N\left(B_{i}(\ell)\right) \simeq F N\left(B_{i}\right)$ for $[n / 2] \leq i \leq n-1$.

For the proof we can realize the sequence $\left(T\left(B_{1}\right), B_{2}, \ldots, B_{n}\right)$ by a knot $k$, using the results of $[\mathbf{L . 1}]$. Now just consider the link $\ell=k \sqcup \ell_{n}^{(m-1)}$ which will have the desired properties.

PROPOSITION (4.2.4). $\ell: \bigcup_{i=1}^{m} K_{i}^{n} \rightarrow S^{n+2}$ is a link of type $i:\{1,2, \ldots, m\}$ $\rightarrow\{1,2, \ldots, r\}$ if and only if there is a surjective homomorphism $\phi_{1}: \pi_{1}(X(\ell)) \rightarrow$ $\left|a_{1}, a_{2}, \ldots, a_{r}\right|$ such the meridional element corresponding to the component $K_{j}$ is sent to $a_{i(j)}$ for $j=1,2, \ldots, m$.

For the proof, just adapt the proof of $[\mathbf{G}]$ for the case of boundary links.

(4.3) Links of two components. For two component links, the universal abelian covering $\tilde{X}(\ell)$ is a covering of $\hat{X}(\ell)$ with group of transformation $Z$. There is an exact sequence of $\Lambda_{2}$-modules $\left(\Lambda_{2}=Z\left[x, y, x^{-1}, y^{-1}\right]\right), \cdots \rightarrow H_{i}(\tilde{X}(\ell)) \stackrel{(x-y)}{\rightarrow}$ $H_{i}(\tilde{X}(\ell)) \rightarrow H_{i}(\hat{X}(\ell)) \rightarrow \cdots$.

As an application of this consider the example given by Hillman in $[\mathbf{H}]$ of a two component link with $H_{1}(\tilde{X}) \simeq \Lambda_{2} \simeq H_{2}(\tilde{X})$ which is not a boundary link. Using the above sequence, we get for this link $H_{1}(\hat{X}) \simeq \Lambda \simeq H_{2}(\hat{X})$ which shows that having a free summand on $H_{1}(\hat{X})$ is not a characteristic of boundary links.

\section{BIBLIOGRAPHY}

[B.1] N. Bourbaki, Éléments de mathématiques, XXVII, Algèbre Commutative, Hermann, Paris, 1968.

[B.2] J. L. Bayley, Alexander invariants of links, Ph.D. Thesis, Univ. of British Columbia, 1977.

[C.E.] S. Eilemberg and H. Cartan, Homological algebra, Princeton Univ. Press, Princeton, N.J., 1956.

[G] M. A. Gutiérrez, Links of codimension two, Revista Colombiana de Matemáticas, 1970.

[H.1] J. A. Hillman, A link with Alexander module free, which is not a homotopy boundary link, J. Pure Appl. Algebra 20 (1981), 1-5.

[H.2] , Alexander ideals of links, Lecture Notes in Math., vol. 895, Springer-Verlag, Berlin and New York, 1981. 
[K] M. A. Kervaire, On higher dimensional knots, Symposium Marston Morse, Princeton, N.J., 1965.

[L.1] J. Levine, Knot modules. I, Trans. Amer. Math. Soc. 229 (1977), 1-50.

[L.2] _ The module of a 2-component link (preprint).

[L.3] _ Unknotting spheres in codimension two, Topology 4 (1965), 9-16.

[L.4] _ $\ldots$ A survey of results concerning the embedding of polyhedra on manifolds in Euclidean spaces (unpublished).

[M.1] H. Matsumura, Commutative algebra, Benjamin/Cummings, 1980.

[M.2] J. Milnor, A duality theorem for Reidmeister torsion, Ann. of Math. 76 (1962),

[N] L. P. Neuwirth, Knot groups, Ann. of Math. Studies, no. 56, Princeton Univ. Press, 1965.

[R] D. Rolfsen, Knots and links, Publish or Perish, 1976.

[S.1] C. Seshadri, Triviality of vector bundles over the affine space $K^{2}$, Proc. Nat. Acad. Sci. U.S.A. 44 (1958), 456-458.

[S.2] Y. Shinohara and D. W. Summers, Homotopy Invariants of cyclic coverings with applications to links, Trans. Amer. Math. Soc. 163 (1972), 101-120.

[T] H. Trotter, On S-equivalence of Seifert matrices, Invent. Math. 20 (1973), 173-207.

[V.1] W. V. Vasconcelos, On local and stable cancelation, An. Acad. Brasil. de Ciênc. 37

[V.2] E. H. van Kampen, Komplexe in euklidischen Raümen, Abh. Math. Sem. Univ. Hamburg 9 (1932), 72-78.

Departamento de matemática, ICMSC, USP, 13.560 São Carlos, Brasil 